\title{
PHOTONEUTRON CROSS SECTIONS FOR BISMUTH-209
}

\author{
Ph.D. Thes is Submitted to lowa State University, \\ February, 1973
}

S. M. Kocimski

\section{MASTER}

Ames Laboratory, USAEC

lowa State University

Ames, lowa 50010

Date of Manuscript: February, 1973

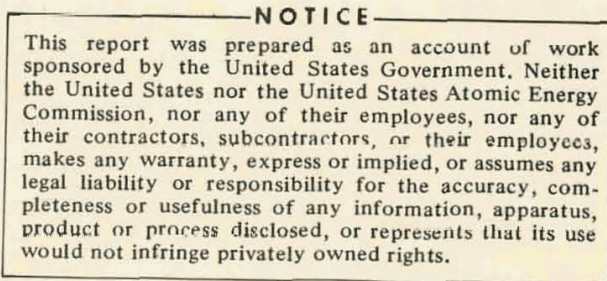

PREPARED FOR THE U. S. ATOMIC ENERGY COMMISSION DIVISION OF RESEARCH UNDER CONTRACT NO. W-7405-eng-82 


\section{DISCLAIMER}

This report was prepared as an account of work sponsored by an agency of the United States Government. Neither the United States Government nor any agency Thereof, nor any of their employees, makes any warranty, express or implied, or assumes any legal liability or responsibility for the accuracy, completeness, or usefulness of any information, apparatus, product, or process disclosed, or represents that its use would not infringe privately owned rights. Reference herein to any specific commercial product, process, or service by trade name, trademark, manufacturer, or otherwise does not necessarily constitute or imply its endorsement, recommendation, or favoring by the United States Government or any agency thereof. The views and opinions of authors expressed herein do not necessarily state or reflect those of the United States Government or any agency thereof. 


\section{DISCLAIMER}

Portions of this document may be illegible in electronic image products. Images are produced from the best available original document. 


This report was prepared as an account of work
sponsored by the United States Government. Neither
the United States nor the United States Atomic
Energy Commission, nor any of their employees, nor
any of their contractors, subcontractors, or their
employees, makes any warranty, express or implied,
or assumes any legal liability or responsibility for the
accuracy, completeness or usefulness of any
information, apparatus, product or process disclosed,
or represents that its use would not infringe privately
owned rights.

Available from: National Technical Information Service Department $A$

Springfield, VA 22151

Price: Microfiche $\$ 0.95$ 
Photoneutron Cross Sections for Bismuth-209

by

Stanislaw Maria Kocinski

A Dissertation submitted to the

Graduate Faculty in Partial Pulfillment of

The Requirements for the Degree of

DOCTOB OF PHILOSOPBY

Department: Physics.

Major: Nuclear Physics

Approved:

In Charge of Hajor $\frac{\text { Cook }}{\text { Cork }}$

For the Hajor Departmeut

For the Graduate CoIjege

Iowa State University

of Science and Technology

Ames, Iowa

1973 
TABLE OP CONTENTS

$\begin{array}{lr}\text { ABSTRACT } & \begin{array}{r}\text { Page } \\ \text { INTRODUCTION }\end{array} \\ \text { I. } & 1 \\ \text { II. GBNBRAL THEORETICAL CONSIDERATIONS } & 3\end{array}$

A. Introduction 3

B. Prediotions for the cross sections 4

III. GENERAL EXPERIUENTAL CONSIDERATIONS 8

A. Methods Used in the Measurements of Photonuclear Cross Sections 8

B. The Derelopment of the Equations 9

C. The Least Squares Method 12

D. Corrections for the Beam Intensity Jitter 16

E. Treatwent of the Natural Background 19

F. Conclusions 20

IV. DETAILS OF THE EXPERIMENTAL EQUIPAENT AND PROCEDORE 21

A. Synchrotron 21

B. Beall Monitoring System 23

C. The Neutron Detector 24

1. Description $\quad 24$

2. Principle of operation 25

3. Tests and procedures 27

D. Experimental Arrangement 42

B. Data Acquisition System 47

P. Data Collection Procedure 50

v. DATA REDUCTION IETHOD 52

A. Preparation of Input Data 52 
B. Unfolding the cross section : 53

VI. EXPERIMENTAL RESULTS AND DISCOSSION

A. The Experiaental Results 57

1. The $(y, n)$ cross section: 57

2. The $(\gamma, 2 \mathrm{n})$ cross section 58

3. The $(\gamma, 3 n)$ and $(y, 4 n)$ cross sections 59

B. Discussion 66

C. Summary 68

VII. IITERATORB CITED \& . 71

VII. ACKHOHLEDGENTS

IX. APPENDIXI: $\quad .74$ 
Photoneutron Cross Sections for Bisuuth-209

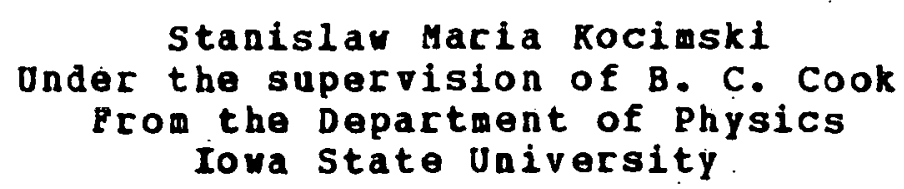

A high efficiency Gd-loaded neutron-sensitive scintillator detector for the photonuclear reactions had been dereloped. The maximum neutron counting efficiency obtained was 82\%. A new method for correcting the results for the ray intensity instability had been developed. A $2.55 \mathrm{~g} / \mathrm{Cm}^{2} \mathrm{Bi}$ tarqet was bombarded with bremstrahlung from the Iowa state University $70 \mathrm{MeV}$ synchrotron. Reaction yields vere obtained at bremsstrahlung end point energies between 9 and $58 \mathrm{MeV}$ at 1 HeV intervals. Subsequently the cross sections for the $(Y, n) \cdot(y, 2 n),(y, 3 n)$ and $(y, 4 n)$ reactions of 209Bi vere extracted from the yield curves by the "least stcucturen technique. The following values were obtained for the sun rules: $s_{0}=3954 \mathrm{mb} \mathrm{MeV}, s_{-1}=264 \mathrm{mb}$ and $s_{-2}=16.6 \mathrm{mb} / \mathrm{Mev}$. For the Lorentz parameters for the total photoneutron cross section the following values were obtained: $\sigma_{0}=565 \mathrm{mb}, E_{x}=13.9$ HeV, and $r=4.5 \mathrm{MeV}$. 


\section{INTBODOCT ION}

A study of nuclear structure is of great importance in verifying the existing theoretical models of nuclei and in providing new data for the construction of new nuclear models and nuclear interactions.

A great portion of the data comes from experimental studies of nuclear reactions. Of these, the reactions caused by y rays are of considerable importance. These reactions are important for the following reasons. The electromagnetic interaction is reasonably vell understood. Also it is relatively weak, wuch weaker than the nuclear interactions. This permits probing the nucleus without disturbing it too qreatly.

of great interest are the studies of simple systems like the magic nuclei. The theoretical predictions for magic nuclei are usually available, making interpretation of the results more meaningful.

Because of recent interest in the region of lead, it was decided in this work to study the cross sections for $209 \mathrm{Bi}$. In addition to measuring the $(\gamma, n)$ cross section it was further decided to measure the cross sections for higher multiplicity reactions like $(\gamma, 2 n),(\gamma, 3 n)$ etc.. and compare them with the predictions of the statistical model. 
In order to achieve this goal, an apparatus had to be developed capable of measuring the $(y, n)$ cross section for nuclei for which the activation wethod as presently used in this laboratory, could not be applied.

The first step in this direction was begun by Jones who built the detector with wich he measured $(\gamma, n)$ and $(y, 2 n)$ cross sections on 19P. However, the deteztor had sereral serious shortcomings wich had to be corrected before any more precise measurements could be made.

Some of the predominant shortcomings were:

1. rather small efficiency for studying $(\gamma, 2 n)$ cross sections

2. high background noise rate

3. intermediate results vere not known soon enough to detect any malfunction of the system.

In the present work these shortcomings vere greatly reduced. The efficiency was very high, the background noise ratio was very low, and data acquisition system gave intermediate results immediately after each run.

subsequently the cross sections for $(\gamma, n),(\gamma, 2 n)$. $(\gamma, 3 n)$ and $(\gamma, 4 n)$ reactions vere measured for $209 \mathrm{Bi}$. 
II. GENERAL THEORETICAL CONSIDERATIONS

\section{a. Introduction}

The dominant feature in the interaction of $Y$ rays with nuclei is the giant dipole resonance. It is the broad peak which appears in the $\gamma$ ray absorption cross sections of nuclei. It appears in all auclei: its energy anj width are slouly: varying functions of the mass number.

The giant dipole resonance has been the subject of Intensive theoretical and experimental investigations. These investigations are not described here in detail since a number of excelient revieu articles have recently been published $(2,3,4,5,6,7,8,9,10,11)$. The main interest of these investigations was to study the structure of the giant dipole resonance. Becently its splitting into components with different isospin (12) has been studied.

Most of these investigations vere limited to the reactions where only one particle is emitted. Very little vork had been done on reactions where more than one particle is eaitted 1ike $(Y, 2 n),(Y, n p),(Y, 3 n)$ etc. The reason is that cross sections for such reactions are very small when compared with $(Y, n)$, and the experimental.difficulties in their neasurement are considerable.

The various theoretical predictions are usualiy based on two models, the older hydrodynamic model, and the newer shell model. 
The shell model calculations are carried out using a particle-hole interaction scheme. Most of these had been made for doubly magic region like $160,40 \mathrm{Ca}$, because of the relative simplicity in the calculations. In the intermediate regions the calculations become too complicated to perform in detail.

Becently a great interest arose in studying the nuclei in the region of lead, $z=82, N=126$. Several aalaulations of the cross sections for $209 \mathrm{pb}$ had been perforned using the shell model. $(13,14,15)$. Recent experiments were performed on $200 \mathrm{pb}$ (16), and earlier on $207 \mathrm{pb}, 208 \mathrm{~Pb}, 209 \mathrm{Bi}$ (17). Theoretical models predict almost no difference in the cross sections for all nuclei in this region.

The goal in this study was to test various theoretical predictions by aeasuring the photonuclear cross sections for $209 \mathrm{Bi}$.

\section{B. Predictions for the cross Sections}

hodel independent calculations lead to several so called sum rules. These rules give the values for the integrated cross sections for the photonuclear reactions.

$$
s_{0}=\int \sigma(E) d E=60 \frac{N Z}{A} B \text { MeV } \times m b
$$

B is an adjustable paraneter of the order of 1. If we set $B=1$ then we have so called classical sur rule of Thomas Reiche and Kuhn: Other two sum rules as quoted by 
Levinger (5):

$$
s_{-1}=\int \frac{\sigma(E)}{E} d E
$$

and

$$
s_{-2}=\int \frac{\sigma(E)}{E^{2}} d E
$$

for $s_{-1}$ Levinger predicts $s_{-1}=\mathrm{CA}^{\frac{4}{3}}$ where $\mathrm{C}=0.35$ ab for an isotropic harmonic oscillator potential, and $c=0.35 \mathrm{mb}$ for finite square vell.

For s-2 Miqdal's calculations (18) predict $s-2=2.25 \mathrm{~A}^{\frac{5}{3}}$ $\mu \mathrm{b} / \mathrm{MeV}$ and Levinger $(5)$ modifies it to $s_{-2}=3.5 \mathrm{a}^{\frac{5}{3}} \mu \mathrm{b} / \mathrm{MeV}$.

The hydrodynamical model of Goldhaber and Teller (19) explains the qiant dipole resonance as due to the relative motions of two interpenetrating incompressible "fluids" of neutrons and protons. The nuclear surface remains fixed and the motion is due to the internal changes in the densities of these fluids. The restoring forces will be proportional to the gradient of the densities. The model predicts the energy of the giant resonance to be $E_{r}=80 \mathrm{~A}^{-\frac{1}{3}}$ Hev. The width of the resonance is due to the number of different modes of vibrations. Since no surface vibrations are included, the only excitations are of the electric dipole nature. Shape of such a resonance can be then described by a Lorentz curve:

$$
\sigma(E)=\sigma_{0} \frac{E^{2} \Gamma^{2}}{\left(E^{2}-E_{Y}^{2}\right)^{2}+E^{2} \Gamma^{2}}
$$


where $\Gamma$ is a width of the giant resonance.

The highly excited nucleus formed by the absorption of $\gamma$ ray may noy decay in various ways. For the excitation energies below the particle threshold the decay will take place through the $Y$ ray emission. Above the particle threshold the nucleus ay exit a neutron, proton, two neutrons, etc., depending on the excitation energy. If one considars the region above the two neutrons emission threshold, the aechanis of the reaction can be described as follows: The nucleus emits (or "boils off") the neutron losing part of its excitation energy. If the intermediate state has an excitation energy above the particle enission threshold, this state may decay again by the emission of a neutron. The statistical considerations led Blatt and Weisskopf (20) to the formula for the ratio of $(\gamma, 2 n)$ to $(\gamma, n)+(\gamma, 2 n)$ cross seztlons:

$$
\frac{\sigma_{2 n}}{\sigma_{n}+\sigma_{2 n}}=1-\left(1+\frac{\varepsilon}{\theta}\right) \exp \left(-\frac{\varepsilon}{\theta}\right)
$$

$\varepsilon$ is the excitation energy above the threshold for the $(\gamma, 2 n)$ process and $O$ is the "nuclear temperature" governing the enission of neutrons from the intermediate nucleus. The nuclear tenperature is slowly varying function of the excitation energp of the nucleus, which is the maxinam energy of the outgoing neutrons. Blatt and Meisskopf (20) shov that

$$
\theta=\left(\frac{F}{a}\right)^{\frac{1}{2}}
$$


The quantity a is one of the parameters which determines the density of levels in the nucleus. Blatt and Neisskopf give that density as

$$
W(E)=C \exp \left(2(a E)^{\frac{1}{2}}\right)
$$

where $C$ is another constant $(C=0.01 \mathrm{MeV}-1$ for $A=181$ and $C=0.005$ Mev-1 for $A=231$ ).

The nuclear temperature describes the energy of enitted neutrons. They 111 have Maruellian distribution, and the most probable neutron energy will be $\theta$.

It is expected, however, that some fraction of the reactions vill not go through the intermediate nucleus stage. Instead, the neutrons will be enitted in the diract process. These "direct" neutrons will not conform to Blatt and Weisskopf statistics, therefore correction should be made in the analysis of the experimental results. 
III. GENERAL EXPERIUENTAL CONSIDERATIONS

A. Hethods Used in the Measurements of Photonuclear Cross Sections

one common method used in the measurement of photonuclear cross sections is photo-activation.

After undergoing the photonuclear reaction were one or more neutrons. or protons are emitted, the resultant nucleus is usually left in the $\beta+$ unstable state and decays with some half-life. From the half-life time analysis a measurement can be taken to determine how many reactions took place, and from this information to obtain the cross sections.

This method has been used extensively in this laboratory.

This method is limited, however, to residual nuclei which are $\beta+$ emitters and whose life-time is between few seconds and approximately 30 minutes. Beyond these linits the experiment becomes difficult or impossible.

The closely celated method is half-life analysis of metastable or excited states of residual nucleus (21).

An alternate method to the activation analysis is the direct detection method, where the protons or neutrons are detected directly from photonuclear reactions.

since the photoneution reactions are dominant, and its cross section measurements are the objective of this study. this paper will emphasize the method of measuring the photoneutron cross sections. 
B. The Development of the Equations

Assume for the moment that we have a detector with a neutron counting efficiency $\varepsilon$. We do not assume anything else about the detector. The results of the analysis should give us some requirements for this detector and for its operating conditions.

Let us first consider two extreme situations. If we pass photons one at the time through the sample, and let the detector count the outgoing neutrons, we would be able to calculate directly the ratios of differing neutron autiplicity. If on the other hand we pass a great number of photons through the sample at one burst, the detector would give us only the average number of neutrons for each beam of photons. We then could not extract ratios of different reactions taking place. In practice we have to operate at sone point between these ertreme positions.

Let us then assume that a burst of photons passes through the sample. Let also $R_{1}, B_{2}, B_{3}$, and $R_{4}$ be the average number of ovents per beam burst of $(\gamma, n),(\gamma, 2 n),(\gamma, 3 n)$, and $(\gamma, 4 n)$ nature respectively. The probability that the number of given events ill take place is given by Poisson distribution:

$$
P(j, k)=\frac{R_{k}^{j} \exp \left(-R_{k}\right)}{j l}
$$


$P(f, k)=$ probability that $f$ reactions of $k$ type vill

take place (k type means $(y, k n)$ ).

So if during the period of the beam burst a namber of different roactions can take place, the probability that the numbr of neutrons will be produced is $r_{m^{\prime}}$ were:

$r_{0}=P(0,1) P(0,2) P(0,3) P(0,4)$

$r_{1}=P(1,1) P(0,2) P(0,3) P(0,4)$

$I_{2}=\mathrm{P}(2,1) \mathrm{P}(0,2) \mathrm{P}(0,3) \mathrm{P}(0,4)+\mathrm{P}(0,1) \mathrm{P}(1,2) \mathrm{P}(0,3) \mathrm{P}(0,4)$

etc.

in qeneral

$$
r_{m}=\sum P(i, i) P(j, 2) P(k, 3) P(l, 4)
$$

where the sumation extends for all indices satisfying the relation:

$$
42+3 k+2 j+i-m
$$

If the detector were $100 \%$ efficient $r_{m}$ would be the numbers we observe in practice. To take into account detector efficiency let us calculate the probability of observing $n$ neutrons, $\mathrm{Y}_{\mathbf{n}}$

$$
\begin{aligned}
& Y_{0}=r_{0}+(1-\varepsilon) r_{1}+(1-\varepsilon)^{2} r_{2}+(1-\varepsilon)^{3} r_{3}+\ldots \\
& Y_{1}=\varepsilon r_{1}+2 \varepsilon(1-\varepsilon) r_{2}+\ldots \\
& Y_{2}=\varepsilon^{2} r_{2}+\ldots
\end{aligned}
$$

and in general the formula is

$$
Y_{n}=\sum_{m=n}^{\infty} \varepsilon^{n}(1-\varepsilon)^{m-n} \frac{m !}{(m-n) ! n !} r_{m}
$$


So at this point we have the probability of detecting $n$ neutrons as a function of $R_{1} \ldots R_{4}$ and $\varepsilon$. The above derivation is a generalization of equations developed by Costa $(22)$ and Jones $(1,23)$.

The best way to invert Equation 13 is to use nonlinear least squares fitting. Before ve turn to that, however, let us consider an alternate method given by Goryachev (24). In this method the moments of the probability distributions are first calculated:

$$
\begin{aligned}
\bar{x} & =\sum_{j=0}^{\infty} j Y_{j} \\
\sigma & =\sum_{j=0}^{\infty} j^{2} Y_{j}
\end{aligned}
$$

From these the values of $R_{1}$ and $R_{2}$ are calculated:

$$
\begin{aligned}
& R_{1}=\left(\bar{x}(\varepsilon+1)-\sigma+\bar{x}^{2}\right) / \varepsilon^{2} \\
& R_{2}=\left(\sigma-\bar{x}^{2}-\bar{x}\right) / 2 \varepsilon^{2}
\end{aligned}
$$

This method has the advantage of simplicity in calculations and of being in a closed form. It is felt, hovever, that least squares method will be more accurate, but the cesults of the Goryachev method for the first estimates will still be used. 


\section{The Least Squares Wethod}

In order to solve the Equation 13 for $R_{1}$ through $R_{4}$.. the nonlinear least squares method (xils) was used. The proqraw was witten in the $P L / 1$ language and followad CURPIT routine (25) utilizing the algorithm given by Marquardt (26) . This algorithm combines gradient search with the method of linearizing the fitting function by first order expansion. It permits rapid convergence from point near or far avay from the minimam of $x^{2}$. To speed the convergence even more for the first estimates the results of the Goryachev method were used.

In addition to greater accuracy, the NLLS method can provide the error of the fitting. In fact, the first use of this method was to find the optimal operating conditions for the detector.

Dump data were generated and the fitting program was used to estimate the errors in the $(\gamma, n)$ through $(\gamma, 4 n)$ rates for various values of $\varepsilon$ and beam intensity (represented here as the arerage no. of counts/beda burst). The figures 1 through 3 show the results of this analysis.

Several important features are immediately apparent. First, there exists an optimal beam intensity, it is about 0.3 counts/beam burst.

The second and very important feature is that in order to do meaningful analysis of reactions of higher neutron 
THIS PAGE

\section{WAS INTENTIONALLY LEFT BLANK}


Figure 1. Error in anfolding the $(\gamma, 2 n)$ rate. 
13

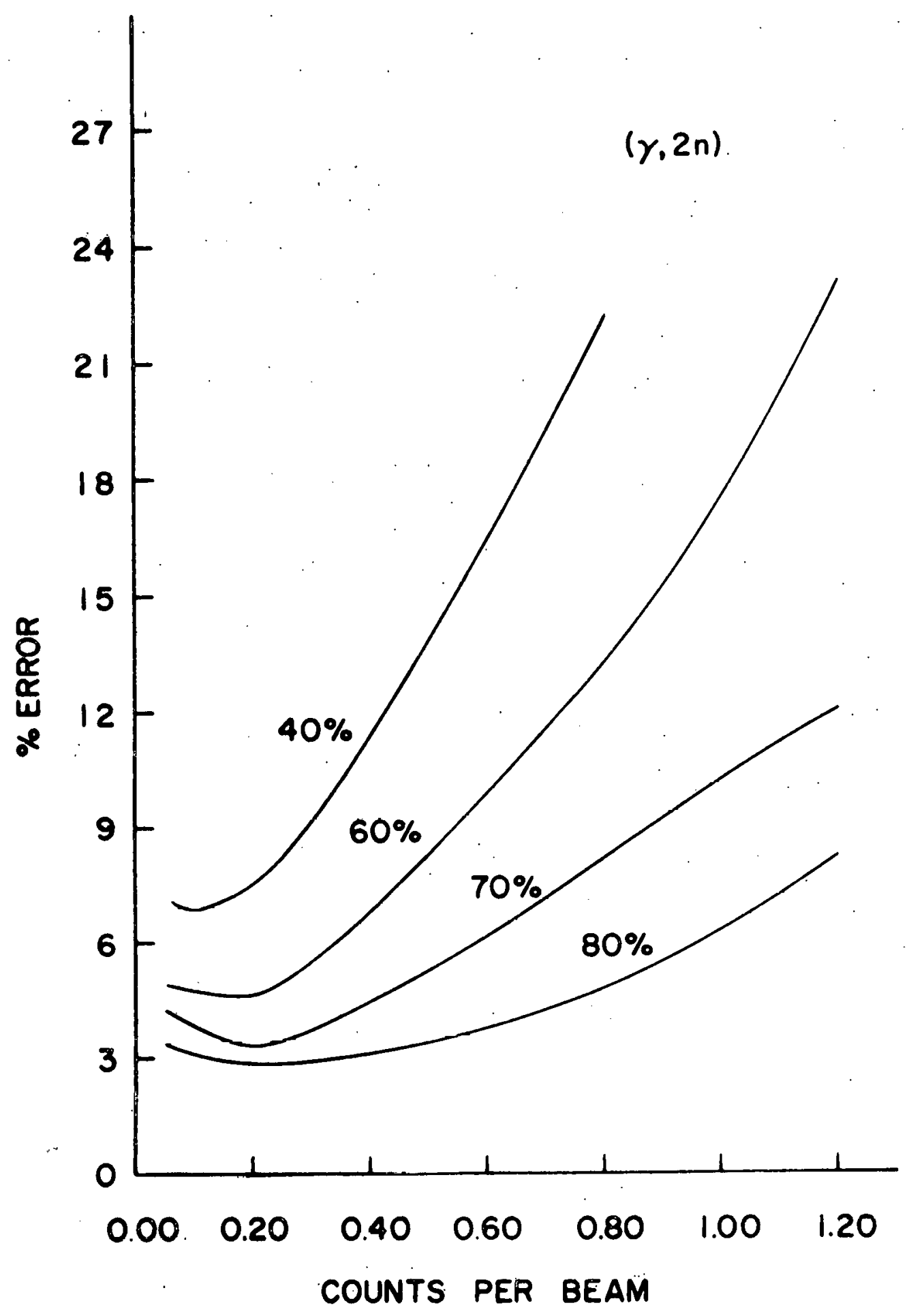


$13 a$

THIS PAGE

\section{WAS INTENTIONALLY \\ LEFT BLANK}


Fiqure 2. Error in unfolding the $\left(\gamma_{0} 3 n\right)$ rate. 
14

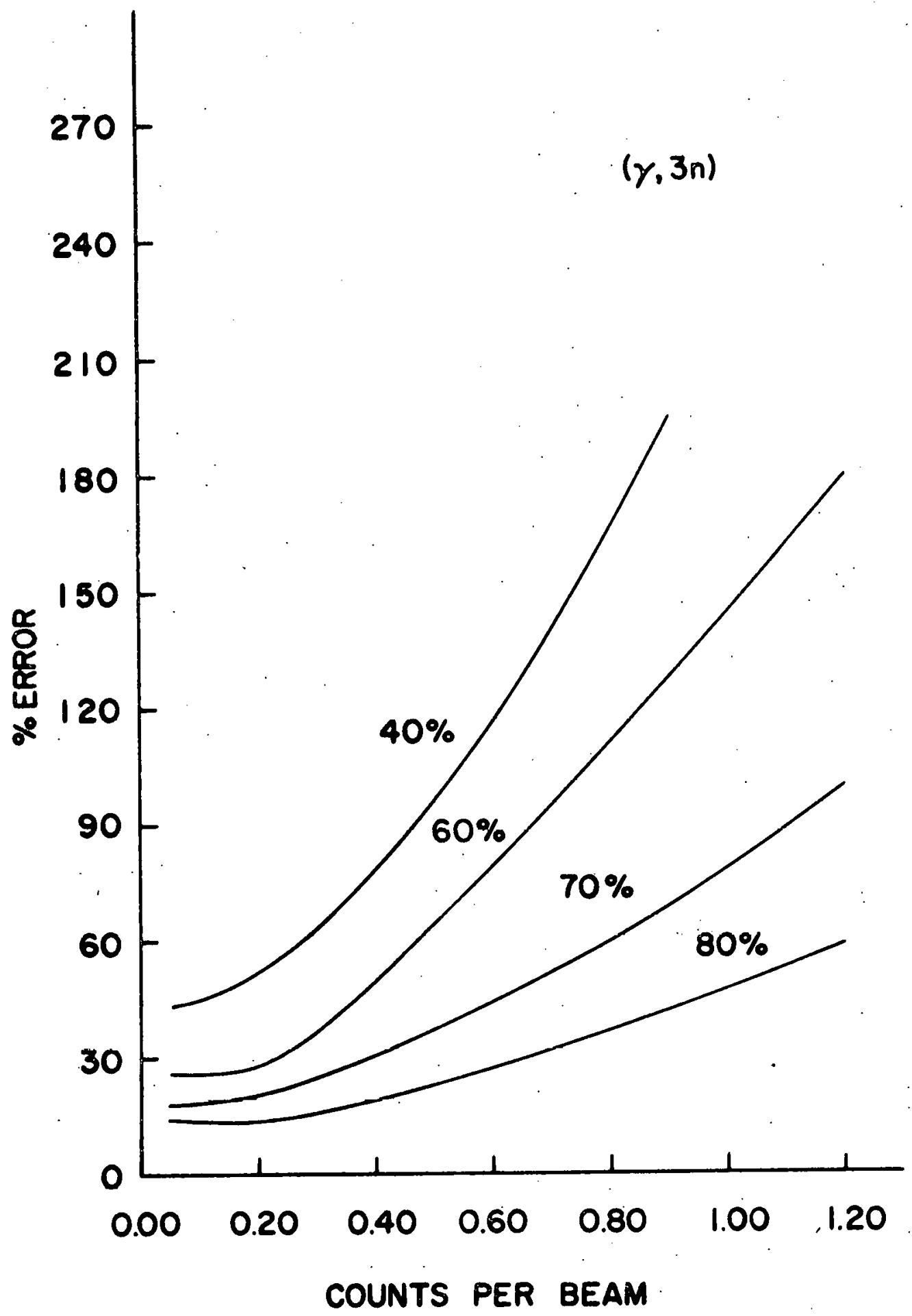


$14 a$

THIS PAGE

WAS INTENTIONALLY

LEFT BLANK 
Figure 3. Error in unfolding the $(\gamma, 4 n)$ rate. 


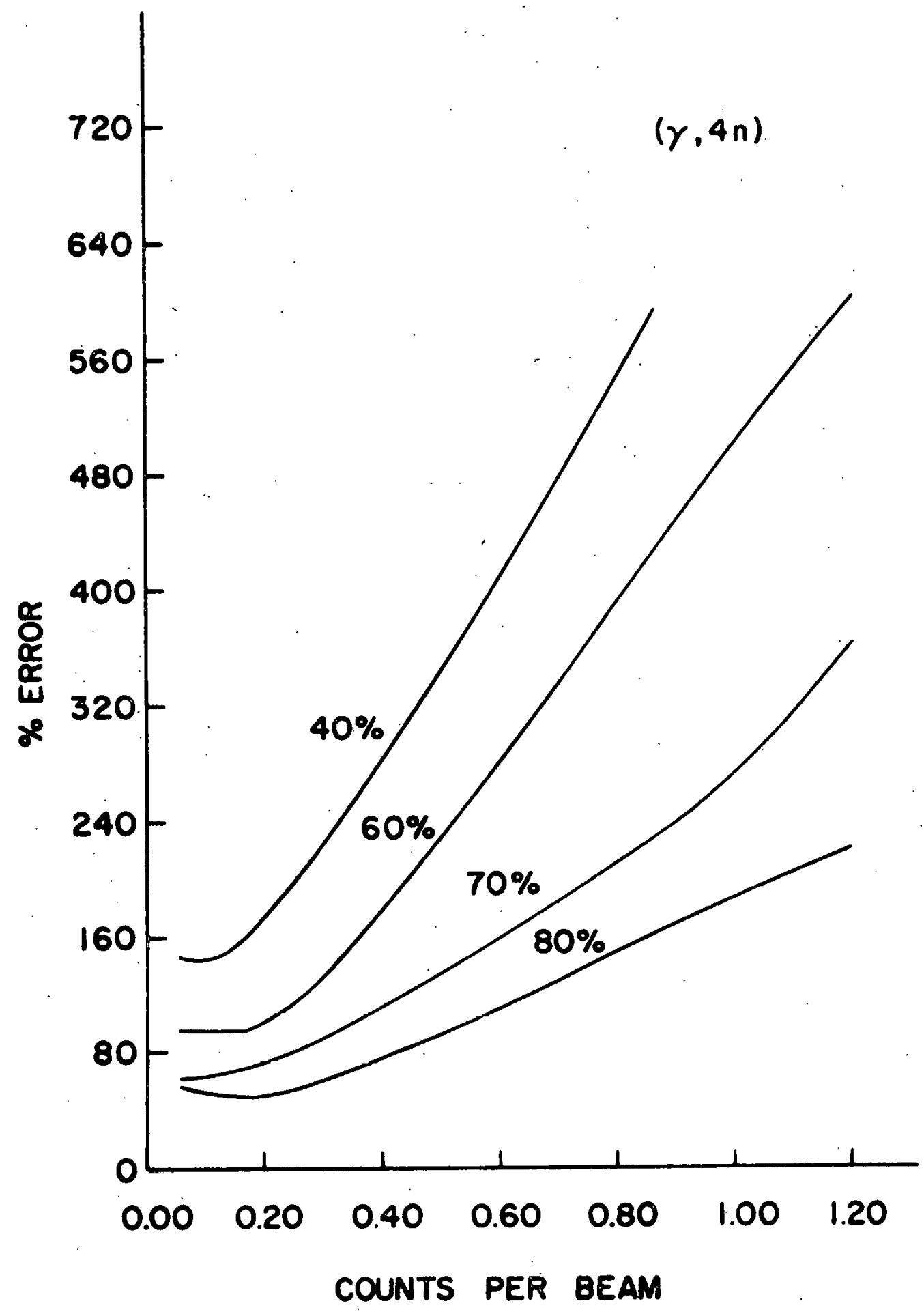


multiplicity, the detector must have high efficiency. Any attempt to do the experiment with detector efficiency of $40 x$ or less would result in measurements with a large possible error.

\section{Corrections for the Beam Intensity Jitter}

All the above analysis has been carried out assuning that beam intensity is constant, i.e.. the number of photons in each beam burst is the same. This is not trua, for despite all efforts beam intensity can not be held more constant than about $15 \%$ to $20 \%$. Thus corrections for the variation rust be made.

Let $Y_{n}$ be some function of $I$, the beam intensity at each pulse. Let us expand $Y_{n}(I)$ in the Taylor series about $I_{0^{\prime}}$ some average intensity.

$$
Y_{n}(I)=Y_{n}\left(I_{0}\right)+\left.\left(I-I_{0}\right) \frac{\partial Y_{n}}{\partial I}\right|_{I=I_{0}}+\left.\frac{1}{2}\left(I-I_{0}\right)^{2} \frac{\partial^{2} Y_{n}}{\partial I^{2}}\right|_{I=I_{0}}+\ldots \quad 18
$$

Let M(I) be the distribution of beam intensities for the run. The averaqe intensity $I_{0}$ vill be:

$$
I_{0}=\frac{\sum I N(I)}{\sum N(I)}=\frac{1}{T} \sum I N(I)
$$

Now the observed probabilities for detecting $k$ neutrons will be the result of "folding" $Y_{k}$ into the beam intensity distribution.

$$
Y_{k}=\frac{\sum N(I) Y_{k}(I)}{\sum N(I)}
$$


substituting the Taylor expansion ve will obtain:

$$
\bar{Y}_{n}=\dot{Y}_{n}\left(I_{0}\right)+\left.\frac{1}{2} \sigma^{2} \frac{\partial^{2} Y_{n}}{\partial I^{2}}\right|_{I=I_{0}}
$$

where

$$
\sigma^{2}=\frac{1}{T} \sum I^{2} N(I)-I_{0}^{2}
$$

So we need to find

$$
\left.\frac{\partial^{2} Y_{n}}{\partial I^{2}}\right|_{I=I_{0}}
$$

becalling the fornula for $y_{n}$ we have:

$$
\frac{\partial^{2} Y_{n}}{\partial I^{2}}=\sum_{m=n}^{\infty} \varepsilon^{n}(1-\varepsilon)^{m-n} \frac{m !}{(m-n) \mid n T} \frac{\partial^{2} r_{m}(I)}{\partial I^{2}}
$$

He have then to express $r_{m}$ as a function of $I$. Becall that $R_{k}$ are the average number of type $k$ reactions per beam pulse. These numbers must be proportional to the intensity, so ve can say that $R_{k}=\bar{R}_{k} I$ where $\bar{k}_{k}$ is the average number of reactions of tppe per unit beam burst.

De can write. nou

$$
r_{m}(I)=\sum \frac{\bar{R}_{j}^{i} \bar{R}_{2}^{j} \bar{R}_{3}^{k} \bar{R}_{4}^{\ell}}{I J J|k| \ell !} e^{-\left(\bar{R}_{1}+\bar{R}_{2}+\bar{R}_{3}+\bar{R}_{4}\right)} e_{I}^{(i+j+k+\ell)}
$$

where again the sumation extends for all values satisfying the relation:

$4 l+3 k+2 j+i=m$ 
After some algebra ve arcive at the formula for

$$
\frac{\partial^{2} r_{m}(I)}{\partial I^{2}}=\vec{r}_{m}(I) \cdot B R(I)
$$

where $\vec{r}_{\mathrm{m}}$ is a pector given by

$$
\vec{r}_{m}=\left(\begin{array}{l}
r_{m} \\
r_{m-1} \\
\dot{r}_{m-8}
\end{array}\right)
$$

Note here that when $a$ is less or equal to eight, the components for these indices are equal to zero.

$\overrightarrow{B R}$ is another vector given by

$$
\overline{B R}=\left(\begin{array}{l}
(S R)^{2} \\
-2 \bar{R}_{1}(S R) \\
-2 \bar{R}_{2}(S R)+\bar{R}_{1}^{2} \\
-2 \bar{R}_{3}(S R)+2 \bar{R}_{1} \bar{R}_{2} \\
-2 \bar{R}_{4}(S R)+2 \bar{R}_{1} \bar{R}_{3}+\bar{R}_{2}^{2} \\
2 \bar{R}_{1} \bar{R}_{4}+2 \bar{R}_{2} \bar{R}_{3} \\
2 \bar{R}_{2} \bar{R}_{4}+\bar{R}_{3}^{2} \\
2 \bar{R}_{3} \bar{R}_{4} \\
\bar{R}_{4}^{2}
\end{array}\right)
$$

where $(S B)=\bar{R}_{1}+\bar{B}_{2}+\bar{B}_{3}+\bar{R}_{4}$

so finally. the formula for the observed probabilities of detecting neutrons in the beam burst, taking into account the bear intensity jitter, is given by

$$
Y_{n}=\sum_{m=n}^{\infty} \varepsilon^{n}(1-\varepsilon)^{m-n} \frac{m !}{(m-n) T n !}\left(r_{m}+\frac{1}{2} \sigma^{2} \vec{r}_{m} \cdot \overrightarrow{B R}\right)
$$


This is the formula to which ve vill apply non-linear least squares fitting to obtain $R_{1}$ through $B_{4^{*}}$

E. Treatment of the Natural Background

Before the measured values of $Y_{n}$ will be used in the vLs fitting, corrections for the natural background have to be made.

If the average numer of counts of natural background is $R_{b g}$ then the probability of detecting $j$ counts due to it in the counting interval, is given also by the poisson distribution. Let $P_{0}, P_{1}, P_{2}, \ldots P_{j}$ be probabilities of detecting 0.1.2.... counts from the natural background respective$1 \mathbf{1}$

probability then of detecting $l$ counts in the counting interval is given by:

$$
\tilde{Y}_{l}=\sum_{j+k=l} P_{j} \dot{Y}_{k}
$$

This can be written also as a matrix equation:

$$
\overrightarrow{\tilde{Y}}=\underline{\mathbf{P}} \cdot \overrightarrow{\mathbf{Y}}
$$

where

$$
\vec{\Psi}=\left(\begin{array}{c}
Y_{0} \\
Y_{1} \\
\cdot
\end{array}\right) \quad \vec{\Psi}=\left(\begin{array}{l}
\dot{\Psi}_{0} \\
\tilde{Y}_{1} \\
\cdot \\
\cdot
\end{array}\right) \quad \underline{P}=\left(\begin{array}{llll}
P_{0} & 0 & 0 & \ldots \\
P_{1} P_{0} & 0 & 0 & \ldots \\
P_{2} P_{1} P_{0} & 0 & \ldots \\
\ldots & \ldots & \ldots
\end{array}\right)
$$


So $\vec{Y}$ will be given by:

$$
\begin{aligned}
& \vec{Y}=\underline{P}^{-1 \cdot} \cdot \vec{Y} \\
& \text { P. Conclusions }
\end{aligned}
$$

The above discussion suggests the following general requirements for the apparatus for the measurement of photoneutron cross sections of different multlplicities:

1. The photons should enter the sample in bursts of intensity that would produce about 0.3 neutrons per burst. The intensity should be as stable is possible. 2. The neutrons produced by the passage of the beam through the sample should be counted by the high efficiency detector (preferably close to $80 \%$ ), and the recorded number stored, so that at the end of the run the distribution of different multiplicities vili be available for processing.

3. There should be a monitor to record the total number of photons ( total dose) which passed through the sample in the course of each run.

4. There should be another monitor registering the intensity of oacb bean burst as it passes the sample. The distribution of the intensities should be obtained in order to calculate the quantity used in Equation 28. 
IV. DETAILS OF THE EXPERIGENTAL EQOIPAENT

A AD PROCEDURE

In this chapter the description vill be given of ail parts of the system measuring the photoneutron cross sections. The results of the discussion in the previous chapter will help to provide an understanding of the reasons for work done to achieve high performance from certain parts of the system.

\section{A. Synchrotron}

As a source of $Y$ radiation the bremsstrahlung from the ISU Electron Synchrotron was used. The detailed description of the machine was given by Griffin (27) and Anderson (28). and for that reason will not be repeated here.

one of the main disadvantages of using this kind of source is that it does not produce monoenergetic $Y$ rays. Instead, the breasstrahlung has a continuous speztrum of energy up to its maximun energy called the "tip". E is equal to the kinetic energy of the electrons striking the breasstrahlung target. Experimental yield are the result of "folding" toqether the bremsstrahlung spectrum and the reaction cross section $\sigma(E)$, and a special technique has to be used to "unfold" the data. 
The method will be discussed further in the ridata reduction" chapter.

Another disadvantage of this particular machine is its 10 pulse repetition rate. The beạm burst comes about 60 times a second. Becalling now that our optimal operating condition is about 0.3 counts/beam burst, an one hour run would give about 50000 counts. This was considered to be the minimu time which should be spent to collect the data for one energy point. Since this factor could not be changed. care had to be taken to design the experiment in such a way that the possibility of making a aistake during the run was as small as possible. such a mistake would be quite costly. It becomes obvious also, that to have intermediate results following each run would be advantageous. It yould then be possible to detect any malfunctions in the system and necessary corrections could be made. During the set-up of the experiaent it was found that the synchrotron while giving a stable beat at high intensities, was rather unstable when it was tuned to the intensity wich rould qive the desired 0.3 counts/beam burst. After eliminating many of the sources of this instability, like drifts in the R.F. system, H.V. injector system etc.. a servo system was added.

Using the fact that beam intensity is a function of injection time, the servo modified the injection timing de- 
pending on the difference between the current bean intensity and its preset value. This method permitted us to obtain $\cdot$ bea stability within $15 \%$ to $20 \%$.

\section{B. Bean Monitoring System}

Two beam monitoring systems vere used in this experiment. One vas used to measure the total dose of rajiation in the qiven period of time. The other one was used to obtain the distribution of single beam intensities.

As a total dose nonitor, a replica of NBS P2 ionization chamber vas used. The original chamber was calibrated with precision by Pruitt and Domen (29). The total charge collected by the chamber was measured by the vibrating reed electrometer (Cary). The output voltage which vas proportional to the collected charge, vas read by the digital voltmeter (DVM):

As a single bean monitor a plastic scintillator mounted on a photomultiplier tube (PMT) was used. The signal from the PMT was electronically integrated and the resultant siqnal was read by $A D C$ and stored by the computer. This monitor was calibrated aqainst the ionization chambar, and was found linear within $15 \%$ throughout the working range. sone difficulties vere encountered, however, with inteqrating the output from the pur. The bean which lasts for about 1 usec has 10 nsec micro-structure, and for this reason vas very difficult to integrate accurately with the 
existing equipment.

\section{The Neutron Detector}

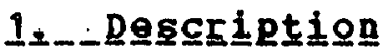

The detector used in this experiment was the improved version of one used by Jones $(1,23)$. It consisted of a cylindrical tank, about 1 long and 1 in diameter, ade out of 1 inch thick aluainium plate. Through the center of the tank passed a stainless steel pipe 8 ca in diameter. Through this pipe the $\gamma$ ray beam passed. The saple target vas placed.in the center of the pipe. Twenty eight 5 inch photomultiplier tubes (EMI 9583B) vere mounted in 4 rous on the cylindrical walls of the tank. The volume of the tank was filled with an organic type liquid scintillator. The picture of the partially open detector is shown in Pigure 4.

The detailed description of the tank is given in (1). 


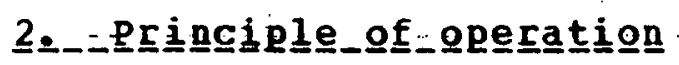

When a high energy neutron enters the tank it scatters on hydrogen nuclei, losing energy. This process known as thermalization lasts until the neutron reaches an energy close to the energy of thermal motion of hydrogen atoms in the liquid. This process takes about $0.3 \mu \mathrm{sec}$. Once the neutron reaches thermal energy it can be capturel by gadolinium present in the tank. The capture process is usually associated with the emission of $\gamma$ rays from the excited final nucleus. These $Y$ rays produce a light flash in the scintillator. Which in turn is detected by the photomultiplier tubes.

Two inportant facts have to be kept in mind while designing such a detector. First, the dimension of the detector should be conparable to or larger than the average radial distance that a neatron will travel before reaching thermal energy. 'For this work Monte Carlo calculations (30) qive this distance to be about $30 \mathrm{~cm}$.

second, the naterial inside the tank should have a high cross section for capture of thermal neutrons. otherwise a large portion of the neutrons will diffuse out of the tank before they will be captured. 
$25 a$

THIS PAGE

\section{WAS INTENTIONALLY LEFT BLANK}


Piqure 4. The giew of the partially open neutron detector. 


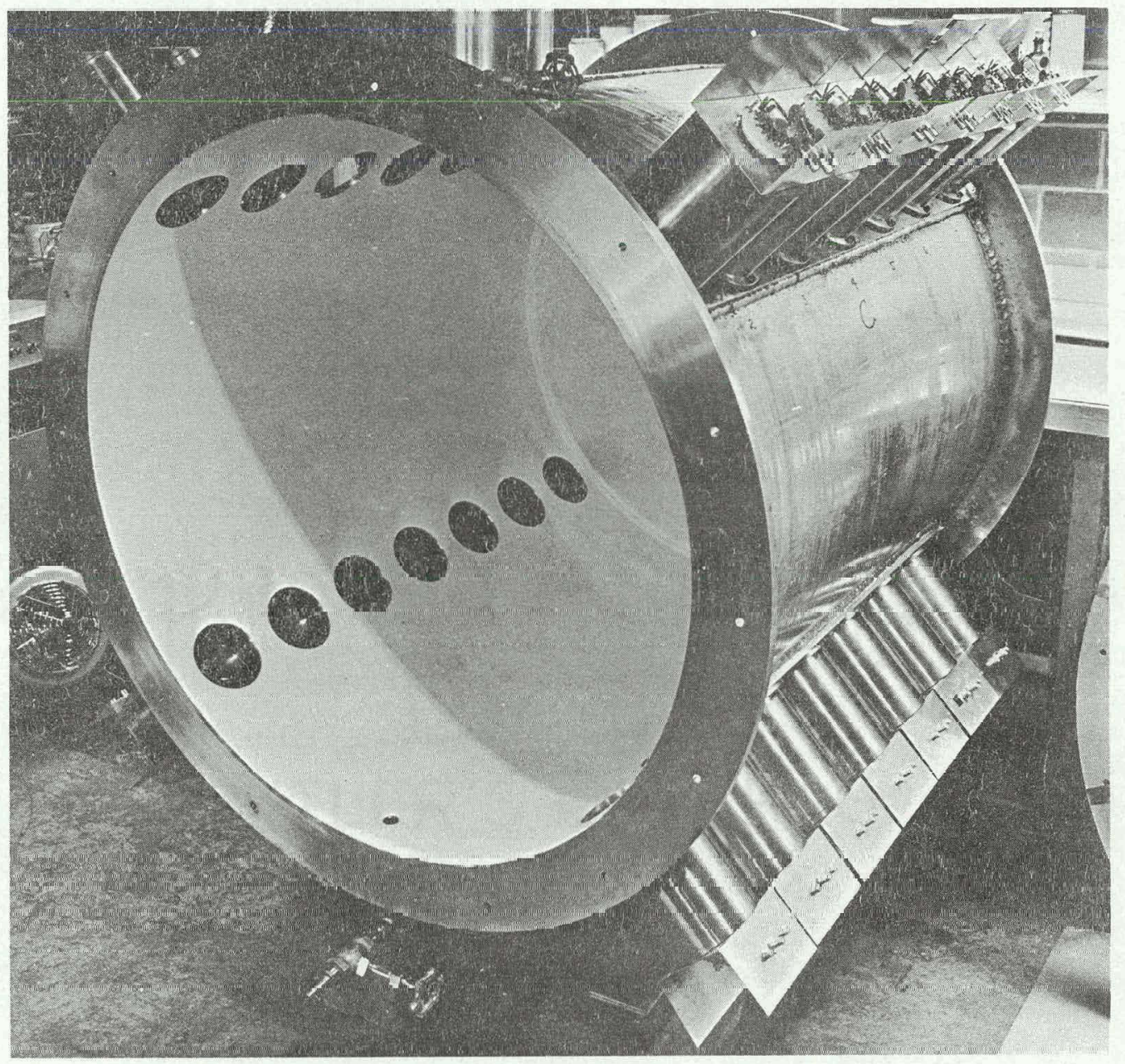




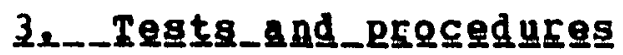

In his original version Jones used a scintillator without the addition of high capture cross section elenents. The neutrons vere captured on hydrogen giving 2.2 Mev $y$ rays. The half-life of neutrons in the tank was 169 usec and efficiency achieved was about 54\%: The natural background associated with his measurements was quite high. The foreground to background ratio was almost $1: 1$.

The obvious way to improve the situation vas to load the scintillator with high capture cross section material. Gadolinium was chosen because of its very high thermal capture cross section (56000 barns) and its availability in this laboratory. Another advantage of using gadolinium was that the capture $y$ rays have an energy of 8.5 thev making them easy to identify.

Some difficulties vere encountered in obtaining a gadolinium conpound which would readily dissolve in an organic solvent. With the help of the Metals Developaent Division of Ames Laboratory two compounds were found: Gd-TBP (Gdtributylo-phosphate) and Gd-2ex-hex (Gd 2 etho hexoate). Gd-TBP was easier to dissolve and therefore vas tried first. However, for sone unknown reasons the entire load turned yellow after 3 months. 
after recovering the gadoliniul another load was uade using Gd-2ex-hex.

The electronic circuits used with the tank vere siailar to those used by Jones. Hoverer, the data acquisition system was completely redesigned. Since PMT's had differing gains, the negative H.V. was supplied to each tube in series with a $500 \mathrm{~K}$ potentlometer. This was done in order to enable us to balance the qains on the phototubes. The procedure is described in detail by Jones (1).

The PMT's vere arranged in two banks of 14 tubes each with adjacent tubes belonging to different banks. Electrical signals from all PMT's in the bank were added together linearly and the output signal was sent to the discriminator. Logic signals from the discriminators from each bank vere then sent to the coincidence circuit. See Figure 5. This arrangement greatly reduced the background noise. Only the signals seen by the both banks simultaneously and above a certain threshold were registered. The thresholds of the discriminators and the width of the pulses (later referred to as "coincidence resolving time", were adjustable.

In order to eliminate background noise from cosmic rays the outputs from Mixers ! and 2 were added and the output was sent to Discriminator 3. If the pulse us above a certain level (in this work, about 9 MeV). the veto signal was gener- 
ated and sent into the coincidence system. This arrangement works like a "windown, that is, it passes a pulse whose height lies between lover and upper levels.

The Discriminator 1 and 2 operated in the mode where the vidth of the output pulse was constant, regardless of the vidth of input pulse. It is important to note that discriminators exist for which the discriminator will repeatedly fire for as long as there is a pulse on the input when the width of the input pulse is larger then the preset width of the output pulse. Multiple triggering, which results in false counts, can be eliminated by setting the output pulse vidth to the maximum width of the input pulse and then clipping the output signal to the desired vidth. This necessarily increases the dead time of the system and thus reduces its efficiency. This problew was encountered by Jones (1), but in this vork a different type of discriminator vas used which did not have this shortconing.

The Discrininator 3 operated in the mode in which the output pulse was set to $200 \mathrm{nsec}$ or the width of the input pulse, whichever was longer. This entire arrangement, which is a part of the data acquisition system, will be referred to in the future as the "pulse selection systen" or PSS and represented as a single block for simplicity. In addition to the logic output an analog output was included wich was the sum of all twenty eight photomultipliers. One of the first 
THIS PAGE

WAS INTENTIONALLY

LEFT BLANK 
Pigure 5. Eiectronic arrangement of the Pulse Selection Systen. 


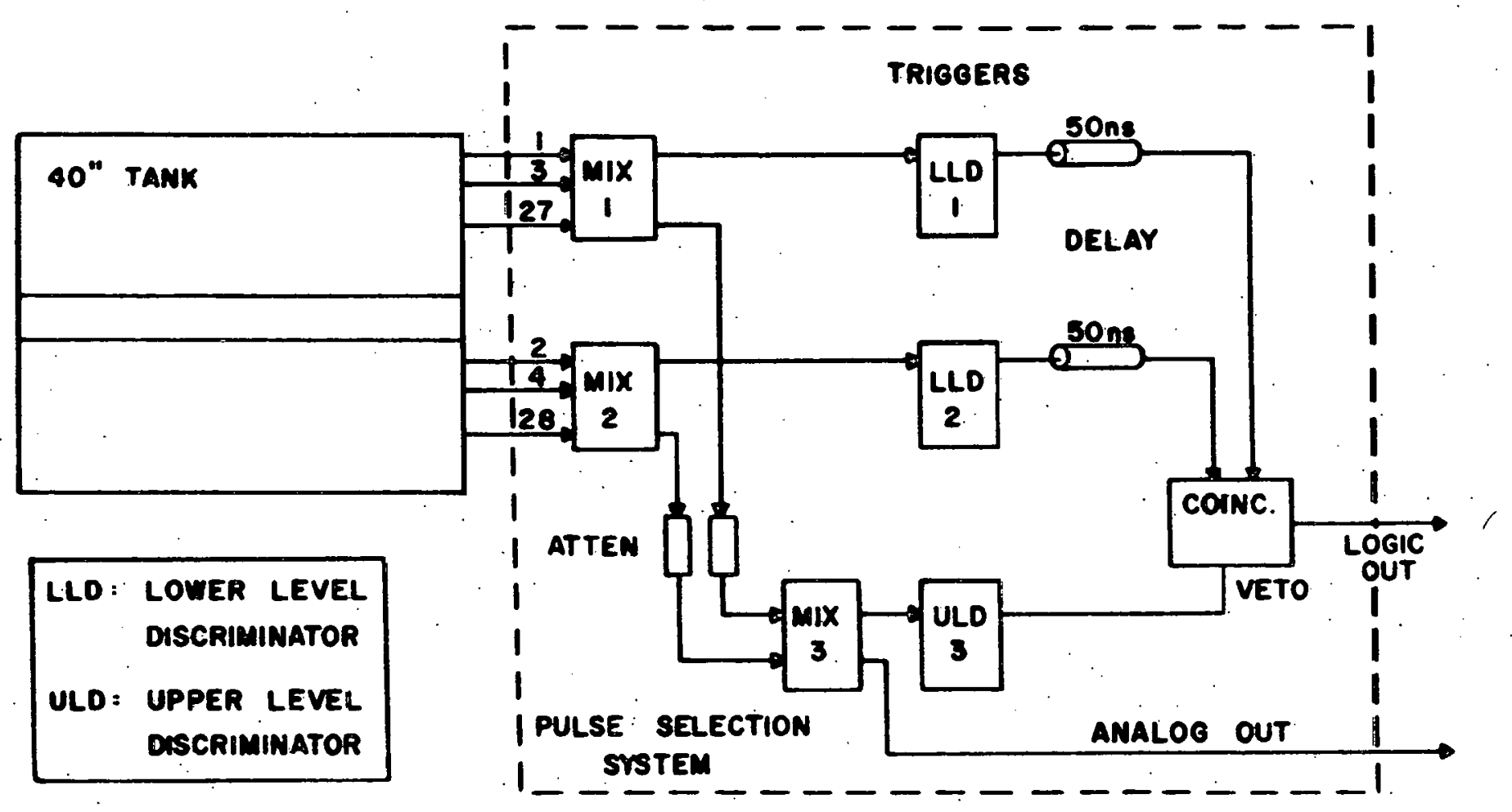




\section{THIS PAGE}

\section{WAS INTENTIONALLY \\ LEFT BLANK}


Figure 6. Electronic arrangement for obtaining the spectra fram different sources. 

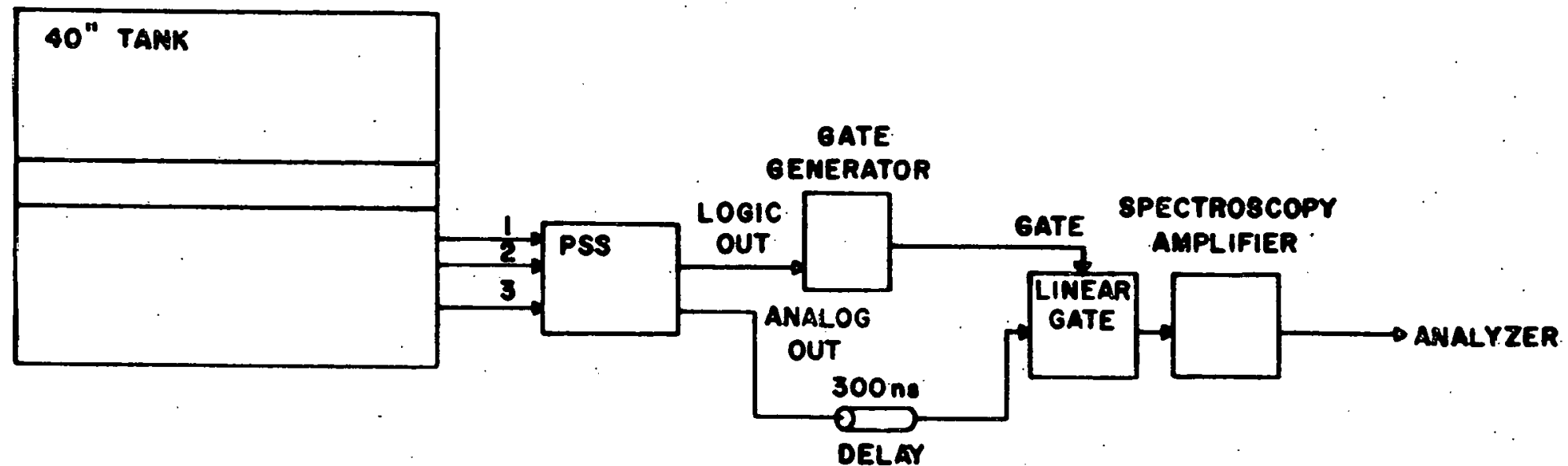

GATE

EMERATOR

ELECTROMICS CONFIGURATION

PSS: PULSE SELECTION SYSTEM 
THIS PAGE

WAS INTENTIONALLY

LEFT BLANK 
Figure 7. Spectra obtained from the neutron detector fron different sources. 

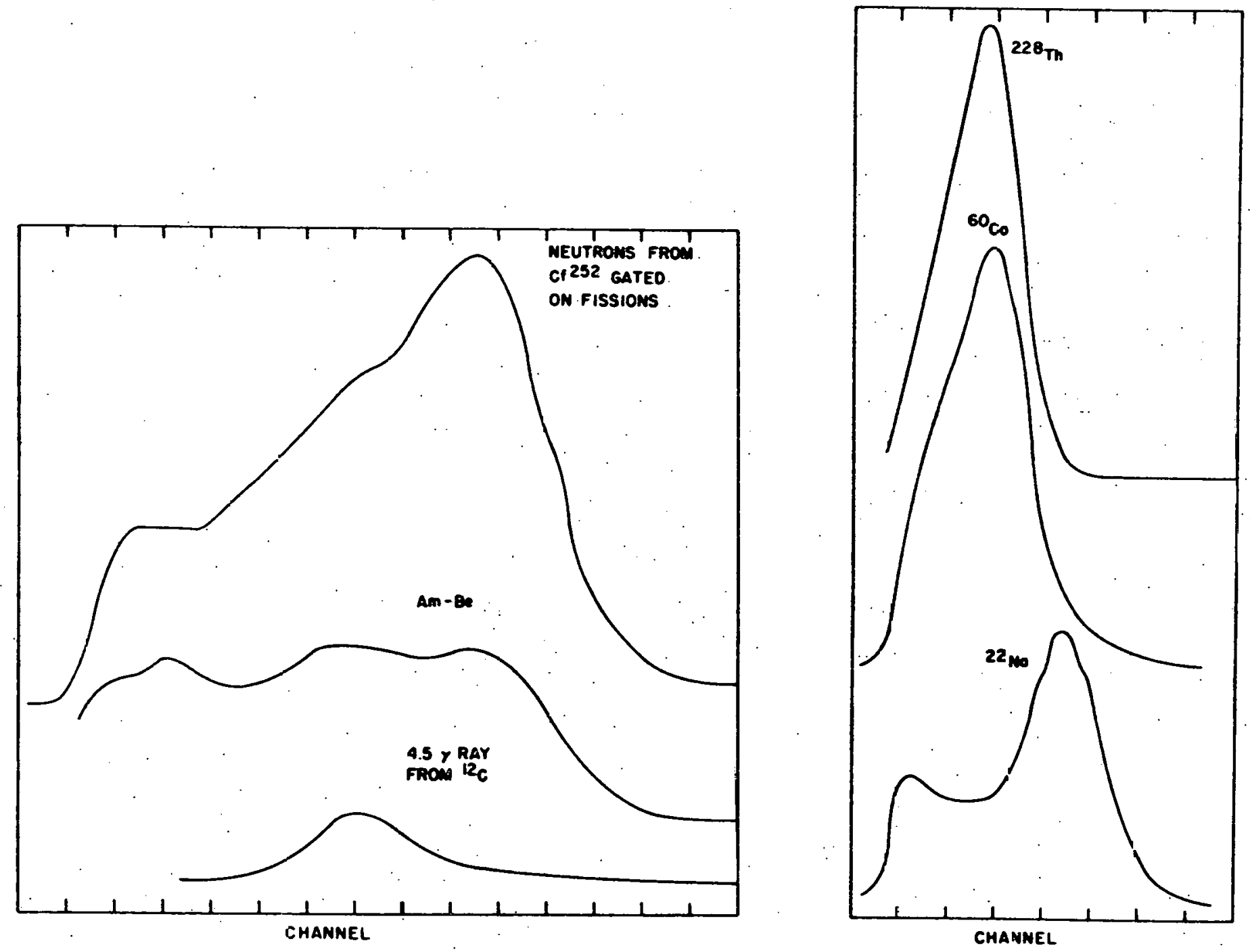
tests performed on the detector was to identify the pulses caused by the neutrons. The arrangement as shown in pigure 6 was used. Thresholds on the discriminators were set to their lowest value and the colncidence resolving time yas 100 nsec.

The spectra for the different sources are given in Pigure 7. It can be seen immediately that the $\gamma$ rays frou the An-Be source are nuch higher in energy than those of $N a$ and Co. They are 8.5 MeV capture $\gamma$ rays from the neutrons. This also shows that it should be rather easy to discriminate out the lower energy background. Separation is much better than in the previous work (1).

In order to measure the half-life of the nentrons in the tank the following procedure was used. An Am-Be source was mounted on a NaI(TI) crystal and inserted inside the beam pipe in the center of the tank. The Am-Be source consists of finely powdered am and Be. Am is the source of a particles which reacting with Be give neutrons:

$4 \mathrm{He}+9 \mathrm{Be}+12 \mathrm{C}+1 \mathrm{n}$

Carbon is a stable isotope, but about $60 x$ of it 15 forned in the excited state, which decays giving a $4.5 \mathrm{MeV}$ r ray. Therefore the detection of 4.5 MeV $\gamma$ ray is an indication that the neutron has been released. This can be used to measure the half life of the neutrons in the tank.

The arrangenent as shoun in Pigure 8 was usad. The 8 MeV $\gamma$ ray pulse was used to start the Time-to-Amplitude 
$33 a$

THIS PAGE

WAS INTENTIONALLY

LEFT BLANK 
Piqure 8. The electronic arrangement to measure the halflife of the neutrons in the tank. 


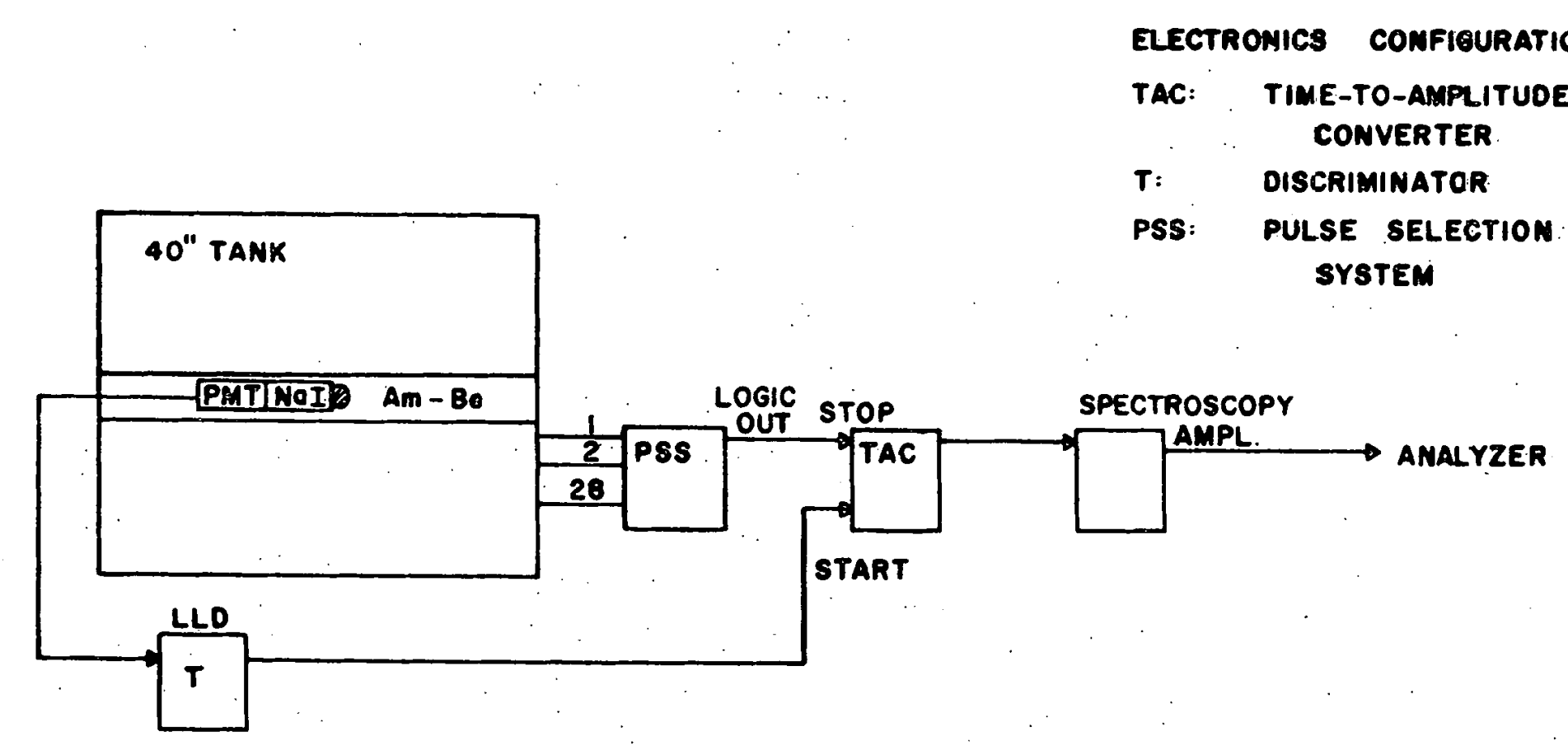


THIS PAGE

\section{WAS INTENTIONALLY LEFT BLANK}


Figure 9. The time spectrum of the neutrons. 


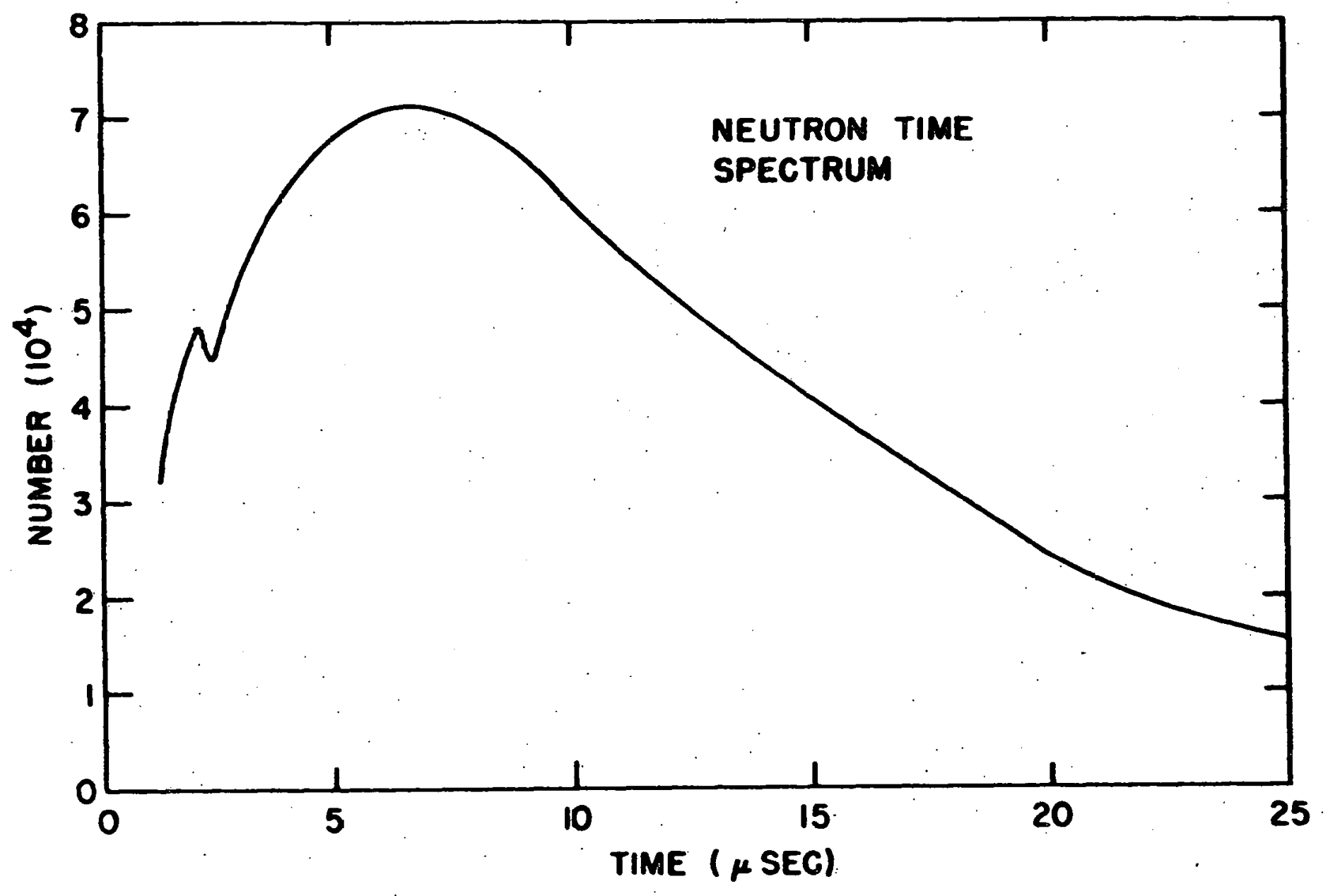

$w$ 
converter, and the signal from the psS stoppel it. The spectrum is shown in Figure 9.

The life time was calculated to be $\tau=5.7$ usec. This figure agrees with the Monte carlo calculations performed for this tank $(30)$

For measurements of efficiency the $252 \mathrm{cf}$ fission source vas used. Californium emits on average 3.275 neutrons while undergoing spontaneous fission (31). Fission events vere detected by using a surface barrier detector. The source was deposited on a flat surface next to the sensitiva area of the detector. Both of them vere placed in the evacuated container wich could be easily inserted into the beam pipe of the tank (see. Figure 10). The experimental circuit is shown in Pigure 11.

The signal from the fission event was used to open the gate. The time length and delay of the gate could be varied. Scaler 1 counted the nuber of gate pulses, while scaler 2 registered the number of neutrons while the gate was open. The efficiency was calculated by dividing counts in scaler 1 by counts in scaler 2, subtracting the natural background, and dividing by the average number of neutrons per fission. Efficiency was then plotted together with BG/Signal ratio for different values of delay, gate width, coincidence resolving time, and the lower level discriminator setting. Plots are given in Figures 12 and 13. 
$36 a$

THIS PAGE

\section{WAS INTENTIONALLY \\ LEFT BLANK}


Figure 10. The $252 \mathrm{Cf}$ source and the fission detector for measuring the neutron counting efficiency. 


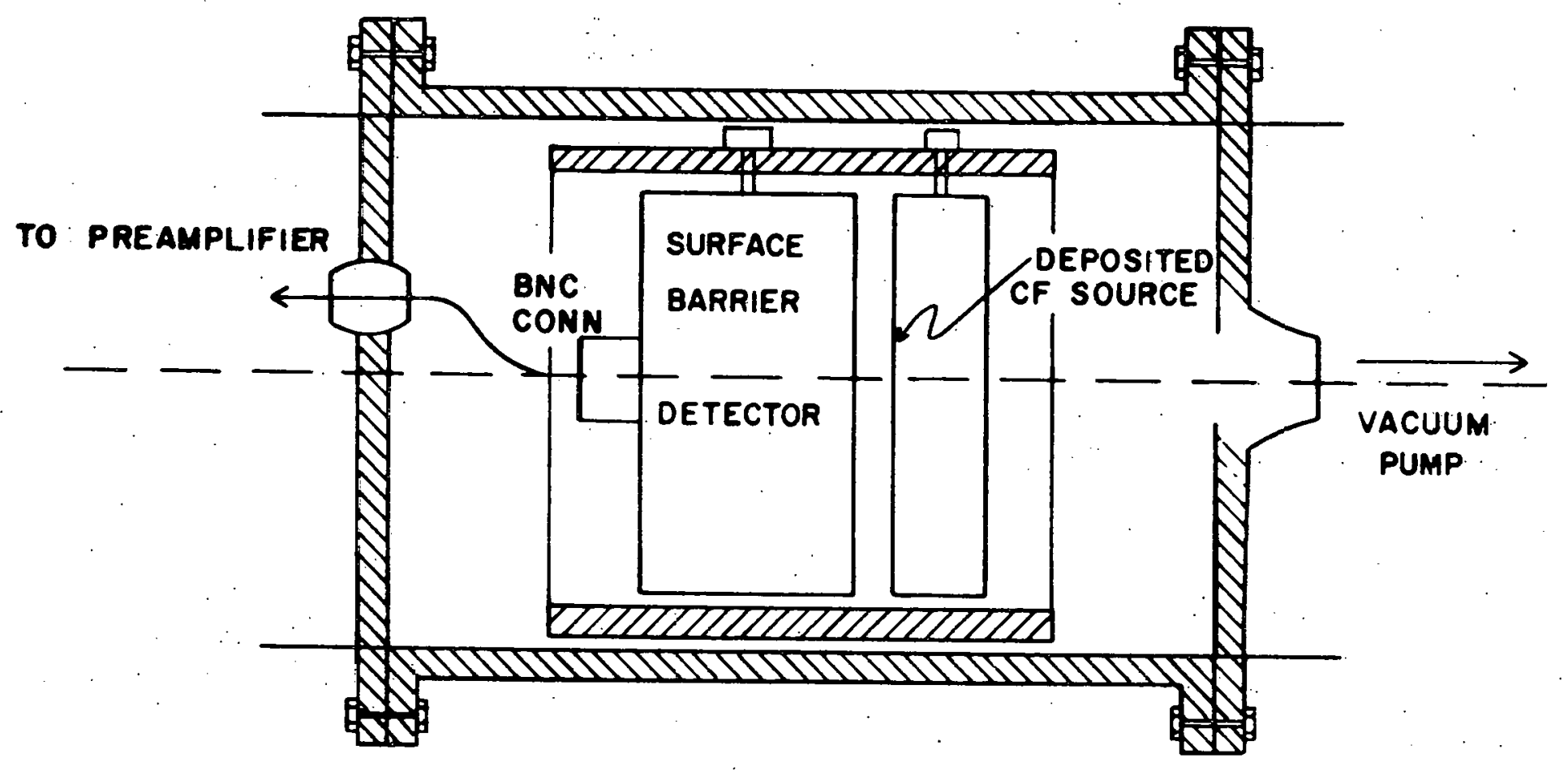


THIS PAGE

\section{WAS INTENTIONALLY LEFT BLANK}


Fiqure 11. The electronic arrangement for measuring the neatron counting efficiency. 


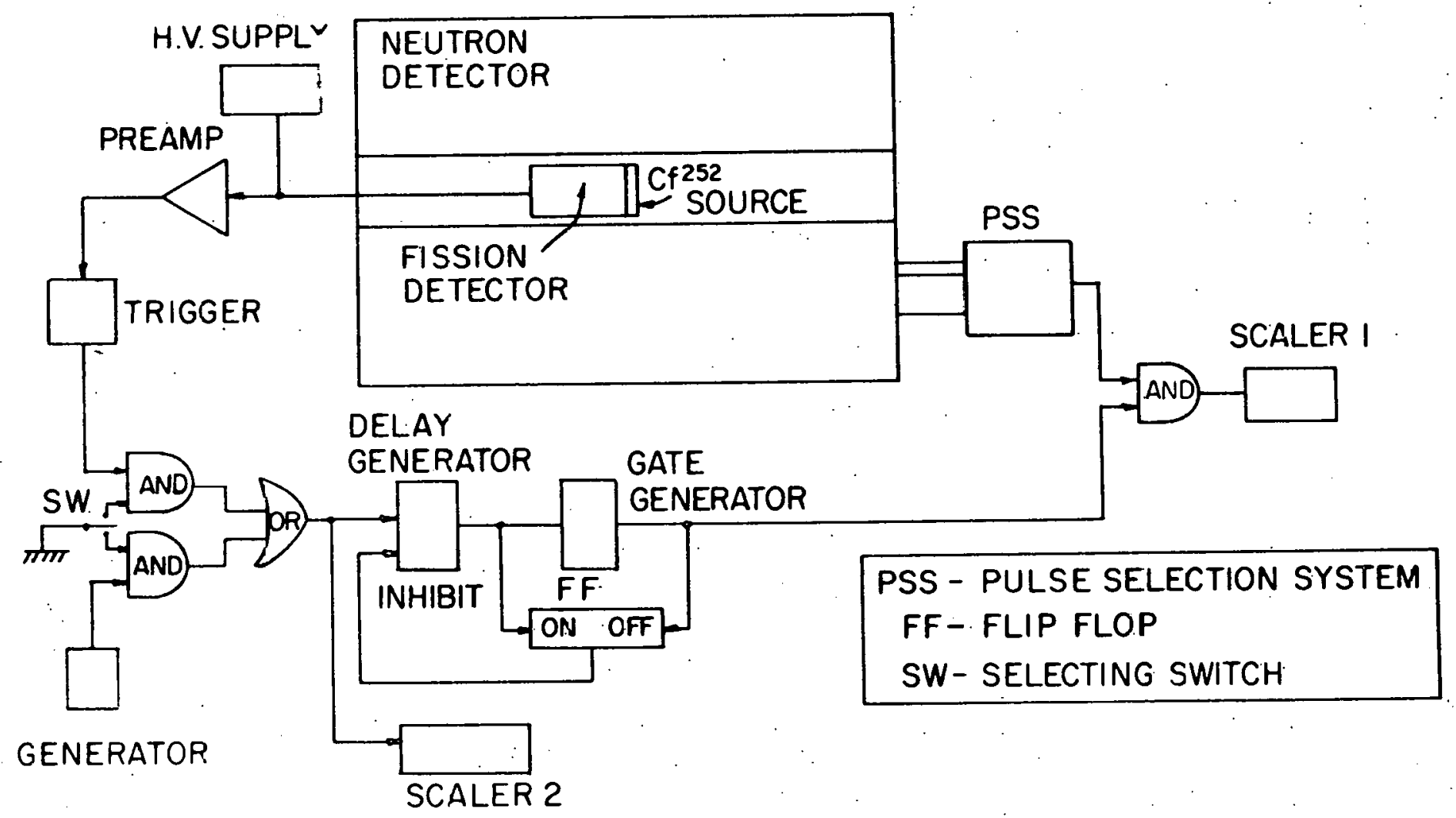


From these we can see that:

1. A delay of less than 2 usec does not change efficiency significantly.

2. While the efficiency increases with the gate length, the BG/signal ratio becomes unfavorabie above 35 . $\mu \mathrm{sec}$.

These trends are independendent of LID setting or coincidence resolving time.

The result (1) is very useful since in the actual run with the bean, some time wust be allowed for the flash from the beam to decay.

It was decided therefore, that for the actual run the count gate vould open 2 psec after the beam passed through, and remain open for 30 usec. The coincidence resolving time and the LID setting were chosen after the tests with the beam, and were set to 25 nsec and 150 m respectively. Since the beam occurs every 17 asec, it yould be economical to measure the background while vaiting for the next beam burst. The timing sequence was set then as follous: (see pigure 14)

After the start of the sequence $T_{0}$ there is a 2 usec vait period for the beam flash to die out. Then the first or "count gaten is generated and the scaler 1 input is opened. After that there is about $5 \mathrm{msec}$ ait and then one hundred 30 usec gates or "backgronnd" gates are generated with 
THIS PAGE

\section{WAS INTENTIONALLY LEFT BLANK}


Fiqure 12. Efficiency and Signal/BG ratio versus the gate vidth. 


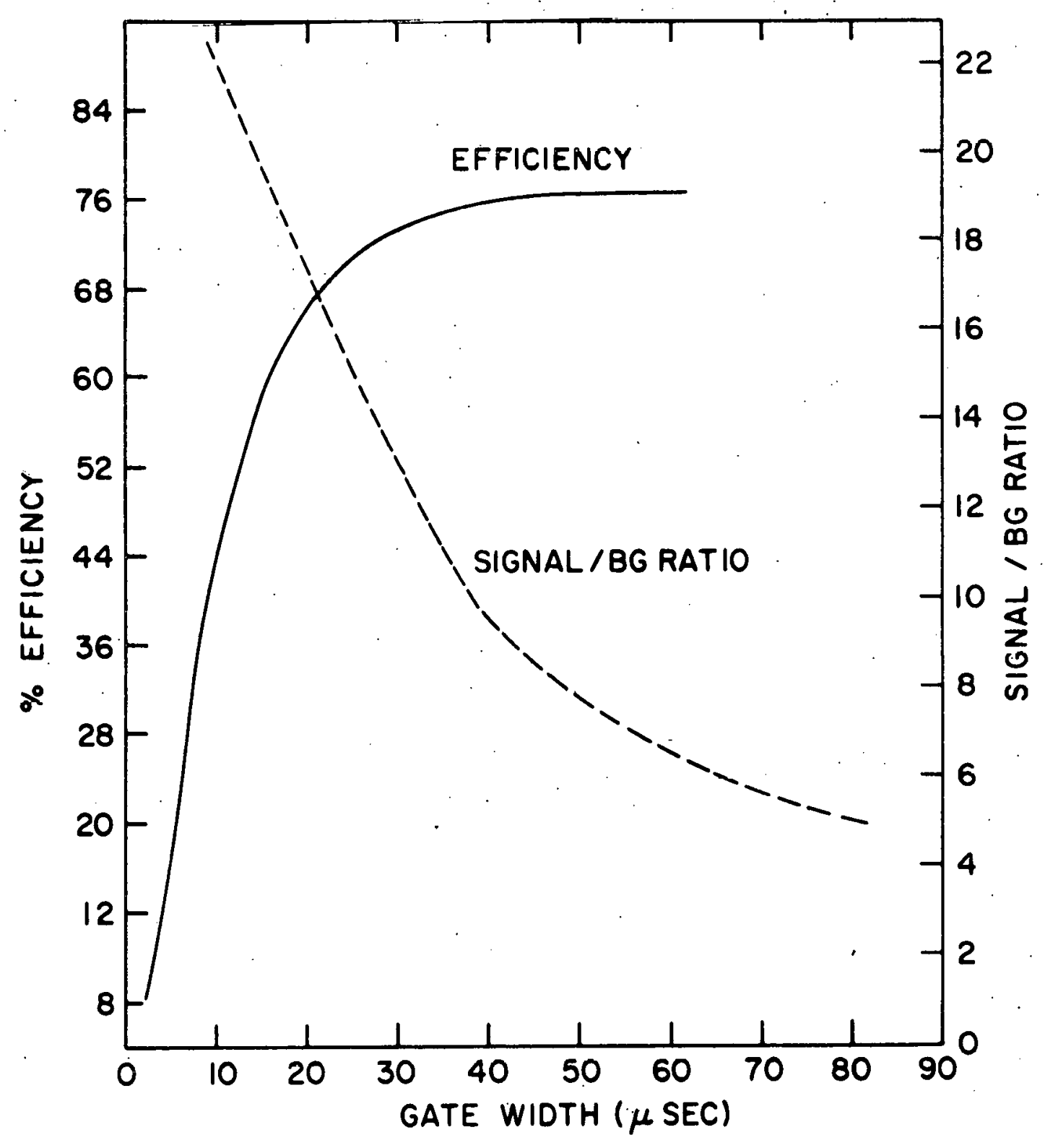


THIS PAGE

\section{WAS INTENTIONALLY LEFT BLANK}


Piqure 13. Efficiency and Signal/BG ratio versus the time delay of gate opening. 


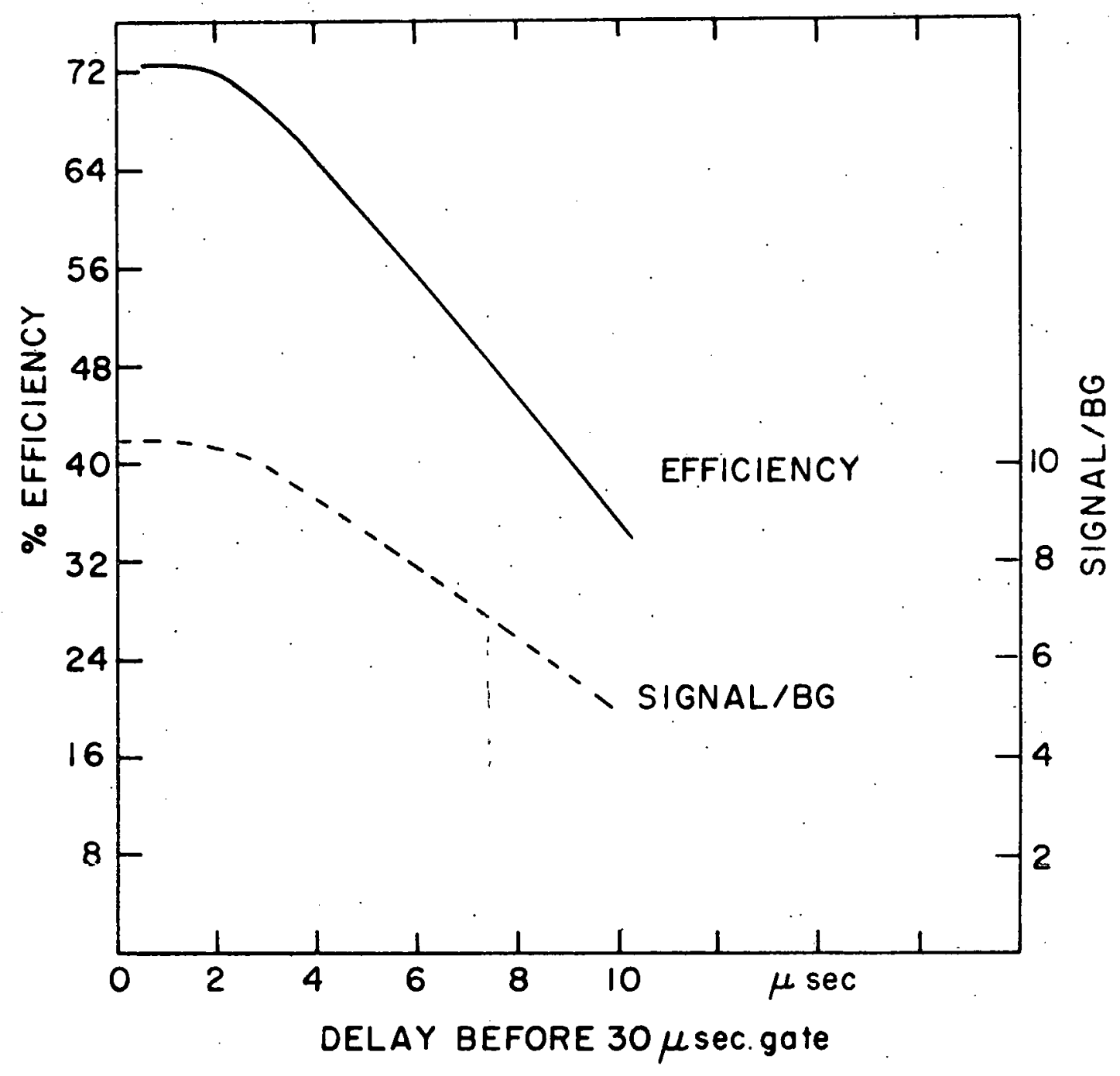


Scaler 2 open. The exact numer of background gates is counted by Scaler 3 .

The electronics arrangement wich accomplishes that is given in Figure 15.

\section{Experimental Arrangement}

The geometry of the experiment is shown in Pigure 16. The bremstrahlung beaw frow the synchrotron was collimated by the tapered tungsten mortar precollimator. Beam was further collimated by the steel collimator on the front wall of the bunker, and then passed through the center pipe of the neutron detector, and two beam monitors.

The tank was enclosed in the concrete bunker to shield It from the natural and wachine generated background. An additional lead wall was built around the tank to further reduce the background. The temperature inside the bunker was kept constant at $16^{\circ} \mathrm{C}$ to retard any chemical reactions which might take place in the scintillator. This also helped to reduce the photonultiplier noise. With the exception of preamplifiers, all the electronics was kept outside the bunker so not to affect the inside temperature.

During the early tests it had been found that the bear qenerates quite significant background (although much smaller than in previous work (1) $)$. This background should not be confused with the natural background which is always present. but rather should be regarded as a contribution to the total 
$42 a$

THIS PAGE

\section{WAS INTENTIONALLY \\ LEFT BLANK}


Figure 14. The timing sequence for the neutron detector. 

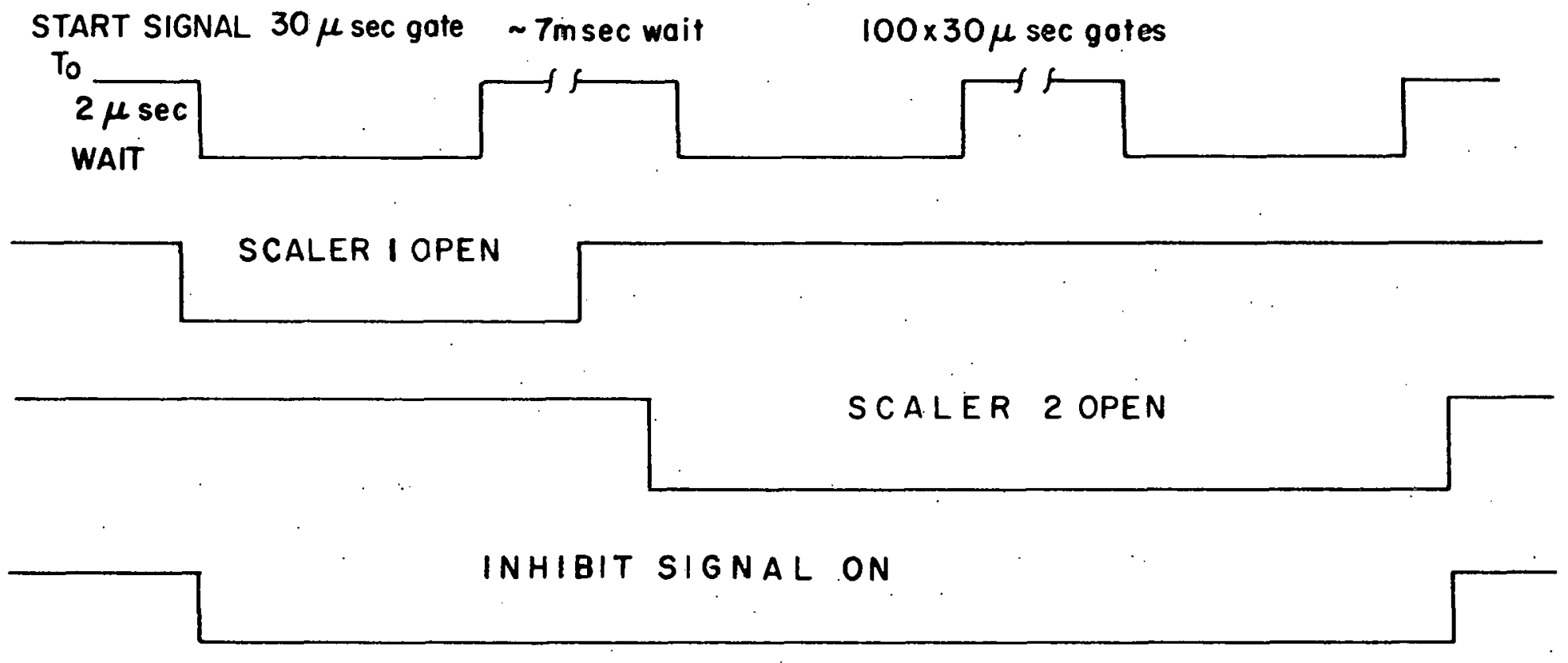


\section{THIS PAGE}

WAS INTENTIONALLY

LEFT BLANK 
Figure 15. The electronic arrangement of the data acquisition syster. 


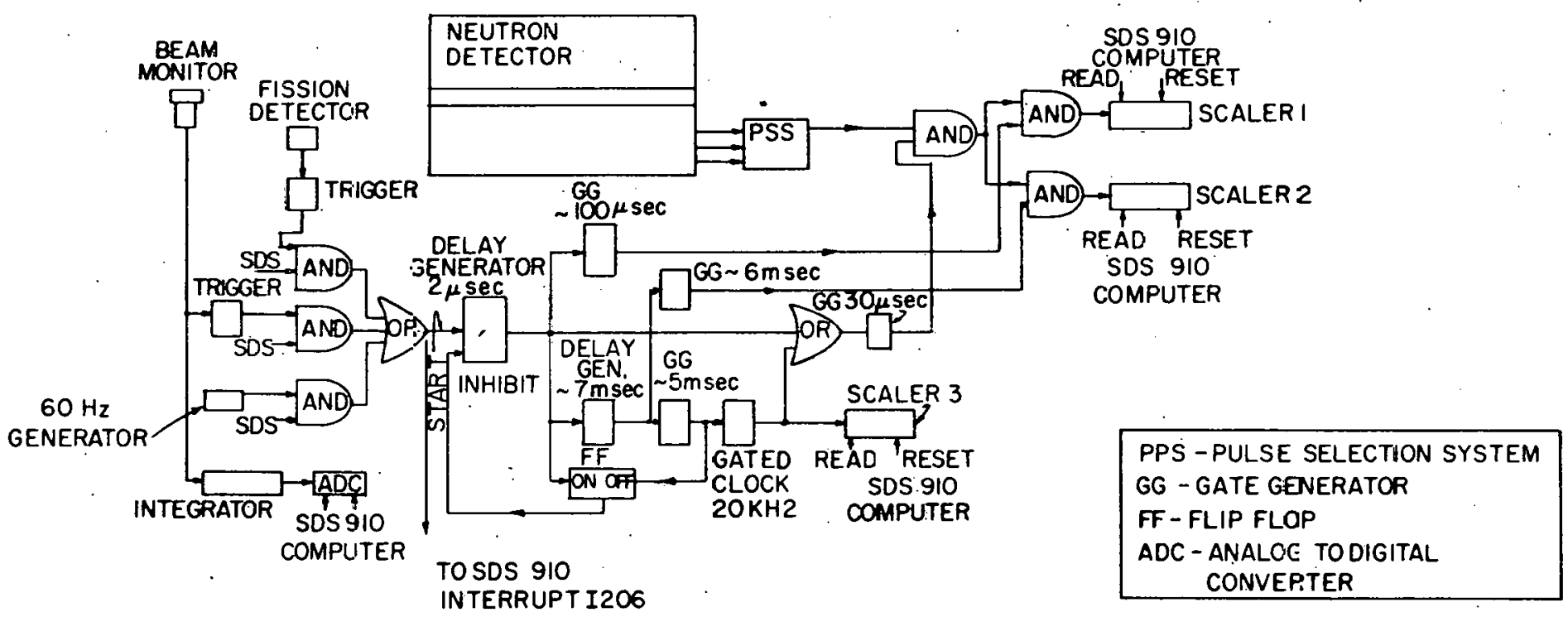


THIS PAGE

WAS INTENTIONALLY

LEFT BLANK 
Pigure 16. The geonetry of the experiment. 


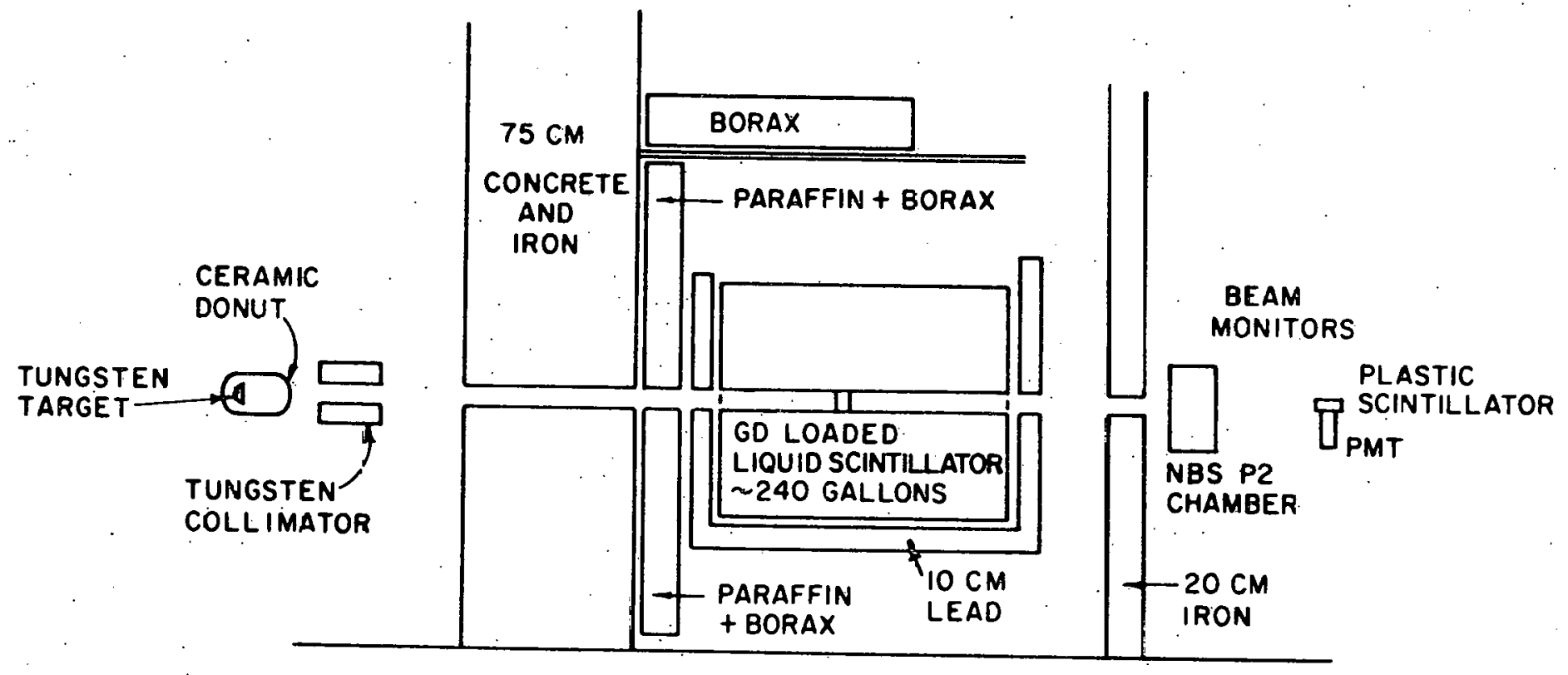


pield from such events as photonuclear reactions in the air or steel pipe inside the tank, neutrons generated in the shielding walls etc. Therefore, two series of measurements should be made, one vith the sample target placed inside the tank, and the other with the target removed. To obtain the offect of the target alone, these rosults should be subtracted.

It is very iaportant also, to monitor for any changes in detector efficiency, NBS P2 chamber response, natural background etc. For these reasons all runs yere divided into four categories called BON, NBBG, STAN, NBBG.

ROd- "regular run" with beam and sample target inside the detector. The tining sequence as started by the signal from the fast bean monitor. At the end of the run, a fit was perforned to obtain values $R_{1}$ and $R_{2}$. which were then plotted on the chart.

MBBG- same as RUN, the only difference being that the target was removed. This run measured nachine generated background.

STAN- standard run. The cf source ras placed inside the tank, and a sr source inside the ionization chamber. The purpose of this run was to check the efficiency of the systen and the response of the ionization chamber.

NBBG- no bean background. No source inside the tank or ionization chamer. The purpose of this run was to measure 
the natural background, the drift of the ionization chamer and its electronics.

Por ROy and RBBG, the spectrum of the bean intensity was also taken.

The results of all the runs yere typed on a teletype, and punched on a paper tape. The tape was thea converted into IBA cards for further processing on an IBH $360 / 65$ compater.

The results of each run included:

1. the distribution $I_{n}$ for the run

2. the total dose of radiation as measured by the P2 chamber

3. the "spectrun" of the beam intensity measured by the plastic scintillator

4. the average rate of natural background

E. Data Acquisition system

While designing the experinent, it was decided to obtain maxinum usage of the on-line computer and eliminate most, if not a1l, of the anual data taking. This eliminated wost of the human errors in this experiment. A1so, it made it possible for only one person to run the entire experinent.

Intermediate results vere then available at the end of each run, waking it possible to detect any malfunction in the system.

A program called NEOTBON was written in real-time assen- 
bly language vhich acconplished this. The detailed description of the NBOTRON program and the SDS 910 computer is outside the scope of this thesis, hoverer, a short description will follow:

The compater had 8192 yords of core storage $(24$ bits per word), an eight wicrosecond cycle time and sixteen priority interrupts. Input/output devices vere a paper tape reader, a paper tape punch, card reader, teletypewriter, a digital display and an oscilloscope display. Data acquisition devices including two digital voltmeters, three scalers, twenty four sense lines and two ADC's vere connected to the computer through the remote interface unit. These devices vere connected to parallei input registers in the remote interface which could be read dicectly into memory.

Sense lines vere connected to the various points of the experimental arrangement. Through them it was possible to test experimentally various conditions (for example; whether the target is inside the tank. position of cf and Sr sources, the scale on CARY-DVM, etc.). Four of the lines vere connected to the control switches, later referred to as "light switches". The status of the sense lines, and CARY-DVH were read continuously by the computer in fixed time intervals. For the operator's convenience, the status of all sense lines was displayed in octal code on the digital display. Devices like scalers and $A D C$ were read asynchronously, using two of 
conputer's sixteen interrupts.

Interrapt I205 or "ADC interrupt" occurred at the end of the conversion process in ADC and signaled that the data vere presented. The computer read the data (representing the bean intensity), calculated the average intensity for the last sixteen beam pulses, displayed it on the oscilioscope, and if the run was in progress stored the data in the memory. Interrupt I206 or "single bean interrupt" caused the computer to read the three scalers and zero then, test the data for internal consistency, calculate the average number of counts in scalier 1 for the last sixteen beam pulses, display the result on the oscilloscope and again if the run was in progress the data vere stored in the memory. These two subroutines were executed every time the intercupt occurred, regardless of the type of the run, or if none of the runs was initiated. In order to start any of the runs, the operator had to select the proper combination of the light switches and then push the button causing 1212 or "start" interrupt. For example, for BuN the target had to be inside the tank, Cf and Sr sources out, Cary on the proper range etc. If all conditions vere satisfied the run was started. The status of the run was displayed on the oscilloscope. The messages included type and current nuber of the run, elapsed time, spectrun of beam intensities, error messages etc. 
If during the course of the run ang error condition ras detected by the computer (like unusually high beam, lack of bea. internal inconsistency of the read data etc.). the run was immediately halted and the proper error message was displayed. After the cause of the ercor was removed, the run could be restarted. The operator could stop the run, terminate it, or completely abort it by selecting the proper conbination of light switches and pushing 1213 or "Stop" interrupt button.

The paraneters, like the duration of the run, the sampling time for reading the dose monitor etc. could be entered from the teletypewriter.

at the end of the run or at its termination, the stored data vere typed and punched on a paper tape. The calcuiations were perforaed to fit the values of $R_{1}$ and $R_{2}$ and the results were typed and punched.

\section{P. Data Collection Procedure}

As was already mentioned, the runs vere divided into four categories. The sequence in wich they vere run as as follows:

1. STAN wich lasted for 10 minutes. A Cf source was placed inside the tank, and sr source inside the ionization chamer. At the end of the run, the efficiency was calculated. Its value was used as an input parameter for the subsequent RON. 
2. Bud which lasted for 60 minutes. a Bi target of thickness of $2.55 \mathrm{~g} / \mathrm{Cm}^{2}$ was placed inside the tank. At the end of the run the rates $R_{1}$ and $R_{2}$ vere calculated and plotted on a chart.

3. MBBG wich followed BON lasted also 60 minutes. The target uas rewored and replaced by the empty target holder. Similarly, the rates $R_{1}$ and $R_{2}$ were calculated and the result plotted.

Following that, the energy was changed and the sequence started from STA again. Every four energy steps an additional RoN was performed at $40 \mathrm{MeV}$ and was followed by NBBG. This was done in order to check further the stability of the systen.

The energies, the order of which was chosen at random, ranged frov 8 weV to 60 weV.in 1 MeV steps. This was done in order to diminish the effect of long time correlations on the yield curve.

The complete yield curve had in principle only one point per energy, but the points wich significantly deviated from the rest of the curve vere rerun. In all, four yield curves vere taken. 


\section{DATA BEDUCTION HETHOD}

The computations performed on the SDS 910 computer were by no means complete. The fit was performed only for $R_{1}$ and $R_{2}$, and corrections for the natural background and bean intensity jitter were not included. Also, at this point only the yield curves vere available.

In this chapter the description will be given of the calculations to obtain the cross sections.

\section{A. Preparation of Input Data}

Uultiplicities vere computed on an IBM $360 / 65$ computer for all four rates. The fit included the correction for the natural background according to Equation 31 and beam intensity jitter according to Equation 28 .

The natural background was plotted for the entice experiment. and its average value of 0.0145 counts/gate was used in the calculations.

The corrections for the beam intensity fitter were incorporated in the nonilinear least squares fitting routine. The resulting yield curres rere then plotted, the deviating points were checked for scientific grounds to be rejected and then the average curve was calculated. The experimental errors vere then calculated for each yield point. 
B. Onfolding the cross section

As was mentioned in an early chapter, the $Y$ ray bea

from a spachrotron is not monoenergetic. The experimental yields were then the result of "foldingi" the cross section with the bremsstrahlung spectran. The expression for the yield per unit onitor response is givon by:

$$
\alpha(E)=\frac{\int N(k, E) \sigma(k) d k}{F(E)}
$$

where $(k, B)$ is the spectruin of the $Y$ rays, given by Schiff (32)

$\sigma(k)$ is the cross section for the photonuclear reaction

$F(E)$ is the response function of the monitor The reduced yield is defined by:

$$
Y(E)=F(E) \alpha(E)=\int N(K, E) \sigma(k) d k
$$

In order to obtain the cross section $\sigma(k)$ ve have to solve the integral Equation 33. Since the quantity $Y$ is obtained experimentally and contains the experimental error, the simple numerical solution can be misleading due to riolent propaqation of ercors when unfolding a matrix with the beharlor of the bremsstrahlung. Numerous methods vere proposed by several authors $(33,34,35)$ to solve this problem. In this work the method of "least structure" developed in this laboratory by B.C. Cook (34) was used. 
In order to describe the method, let as first approriate the integral Bquation 33 by a atrix equation

$$
Y_{i}=\sum_{j=1}^{n} N_{i j} s_{j}
$$

where

and

$$
s_{j}=\frac{1}{\Delta E} \int_{E_{j}}^{E_{j}} \sigma(E) d E
$$

$$
N_{i j}=\int_{E j-\Delta E}^{E_{j}} N\left(E, E_{i}\right) d E
$$

The solution of the equation for the relative cross section is then obtained by multiplying by the inverse matrix. This is the method developed by penfold and Leiss (33). In order to obtain the solution that does not oscillate violently. the smoothing has to be applied, preferably a nonsubjective one. The "least structure" method requires that the cross section be smoothed to a solution consistent with the accuracy of the vield curve. A solution is defined to be consistent with the pield curve accuracy in terms of the statistics $\chi^{2}$, i.e.

$$
x^{2}-\sum_{i=1}^{n} \frac{(j=i}{\left.\sum_{i j}^{n} N_{i j} s_{j}-Y_{i}\right)^{2}}
$$

where $\Delta Y_{1}$ is the error in the field at the ith energy. Solutions are accepted if $x^{2} \leq n$. There are, in fact, an infinite number of solutions to satisfy this condition. The "least structuren selects the "smoothest" set of solutions which 
satisfy the $x^{2}$ condition. It is done by minimizing the so called natructure function $s\left(s_{j}\right)$ defined as:

$$
s\left(s_{j}\right)=\sum_{j=1}^{n-1} P_{j}\left(s_{j+1}-2 s_{j}+s_{j-1}\right)^{2}
$$

which is the weighted sur of the squares of the second differences. ( $P_{f}$ is a weight hich allows smoothing to be applied in a uniform manner.)

The problea of finding the smoothed solution can be written using variational calculus as:

$$
\lambda \delta s\left(s_{j}\right)+\delta \chi^{2}\left(s_{j}\right)=0
$$

where $\lambda$ is a measure of sooothing applied. For fixed $\lambda$ the result of variation is:

$$
\mathbf{Y}_{i}=\left\{\mathbf{N}_{i j}+\lambda\left(\left(\Delta Y_{i}\right)^{2} \delta_{i k} P_{k} \delta_{k q} \bar{N}^{-1} s_{p j}\right)\right\} s_{j}
$$

or

$$
\mathbf{Y}_{i}=M_{i j} \mathbf{s}_{j}
$$

Hence

$$
s_{j}=M_{j i}^{-1} Y_{i}
$$

Purther discussion of the least structure technique is found in $(34)$.

The reduced pields were first calculated froin the averaqe yield curves, taking into account the absorption of 
rays in the target, donut wall. and air. Next, the background (DBBG) curve was subtracted from the Rou curve. The resultanc curve which represented the effect of the target, was then processed by the "least structure" routine (CLSR). 
VI. EXPERIUENTAL RESULTS AND DISCOSSION

The average reduced yield curves obtained by the method described in chapter $V$ were unfolded by the "least structure" procedure to obtain the cross sections. These calculations vere also performed for the yield curves obtained without applying the corrections for the bea intensity fitter.

For the $(\gamma, 3 n)$ and $(y, 4 n)$ reactions the large experimental uncertainties did not allow extraction of differential cross sections. Instead, an estimate for the integrated cross sections up to $58 \mathrm{MeV}$ was obtained.

\section{A. The Experimental Besults}

1. The $\left(\gamma_{2}\right.$ ㄴ $L$ cros

The $(\gamma, n)$ cross section curve, as obtained from the chsB analysis is shown in figure 17. The difference between this curve and the one obtained from the yield without the correction for the beam intensity jitter applied, was not detectable.

The slight negative undershoot of the cross section curve in the region above $19 \mathrm{MeV}$ may be attributed to the effect of the shape of the resolution function. If one assumes that the true cross section at these energies is close to zero, then the negative portion of the resolution function contributes fust the right amount of the negative cross section. (The shape of the resolution function depends on the 
experimental errors of the yield curve, if the errors are large the resolution function tends to be broader and the negative undershoots are larger, if on the other hand, errors go to zero. the resolution function approaches the $\delta$-function shape) .

The integrated cross section value is $2830 \pm 100 \mathrm{mb}$ MeV. Harver et al. (17) using the quasimonochromatic rays obtained the $(\gamma, n)$ cross section curve as shown in Figure 18. His value of integrated cross section is 2970 mbxMeV which is about $30 \%$ lower than from this experiment. The same paper, however. gives an integrated cross section for $200 \mathrm{~Pb}$ which is again about $30 \$$ lover than the result of Veysiere et al. (17). The shape of the $(y, n)$ cross section curve of this experiment agrees with the result of Harvey et al. rather well. The cross section vas fitted with a Lorentz curve corrected for the barrier transmission coefficient. The fit was performed for the points below 14.5 HeV, the $(\gamma, 2 n)$ reaction threshold. The values obtained for the Lorentz parameters vere: $\sigma_{0}=565 \mathrm{mb}, \mathrm{B}_{\mathrm{r}}=13.9 \mathrm{HeV}, \Gamma=4.50 \mathrm{HeV}$.

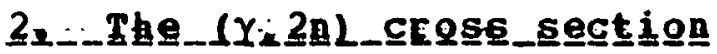

Two cross section curves were deduced for the $(Y, 2 n)$ reaction from the reduced yield functions with and without corrections for the beam instabilities. In contrast to the $(\gamma, n)$ case, differences between these two curres can be seen. The "uncorrected" curve is shown in Figure 19 and the "cor- 
rected" one in Figure 20.

The effect of the correction for the bean intensity jitter on the $(Y, 2 n)$ cross section is quite large and also can be seen in the yield curves. Howerer, wh added uncertainty in establishing the value of $\sigma$ of the beam intensity spread, no valid conclusion can be wade as to the shape of the resultant curve. This is because the resolution function is very broad and severely distorts the shape of the cross section curve. It is also impossible to make any valid comparison with the statistical model predictions.

The Integrated cross section value was $732 \pm 30 \mathrm{ab}$ HeV. For comparison the $(Y, 2 n)$ cross section curve as obtained by Harvey et al. is shown in Pigure 21. The value of the inteqrated cross section in that work vas $760 \mathrm{mb} \times \mathrm{HeV}$, in good aqreenent with present work.

3. The

The large experimental uncertainties in the yield curves made it impossible to obtain the differential cross sections for $(\gamma, 3 n)$ and $(\gamma, 4 n)$ reactions from CLSR routine. However. the values of the integrated cross sections up to 58 lev rere calculated.

P1rst, Pentold-Leiss analysis was performed, which was equivalent to setting $\lambda=0$ in the cLsR routine. The resulting values were added together to obtain the integrated cross section. Despite the large scatter of the points, the aver- 
THIS PAGE WAS INTENTIONALLY LEFT BLANK 


$$
-
$$




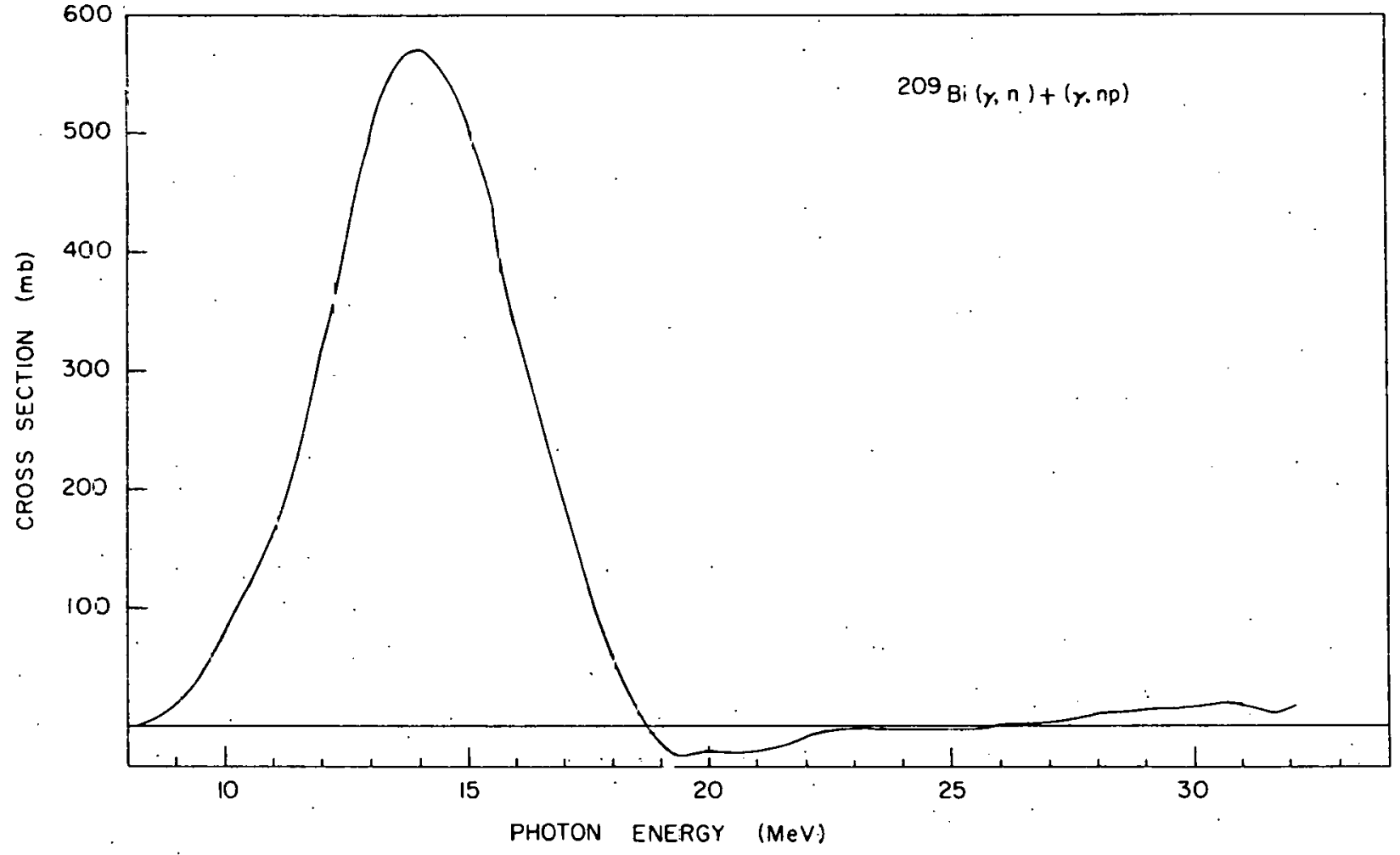


THIS PAGE

WAS INTENTIONALLY

LEFT BLANK 
Fiqure 18. The $(\gamma, n)$ cross section curve as obtained by Harvey et al. (17). 


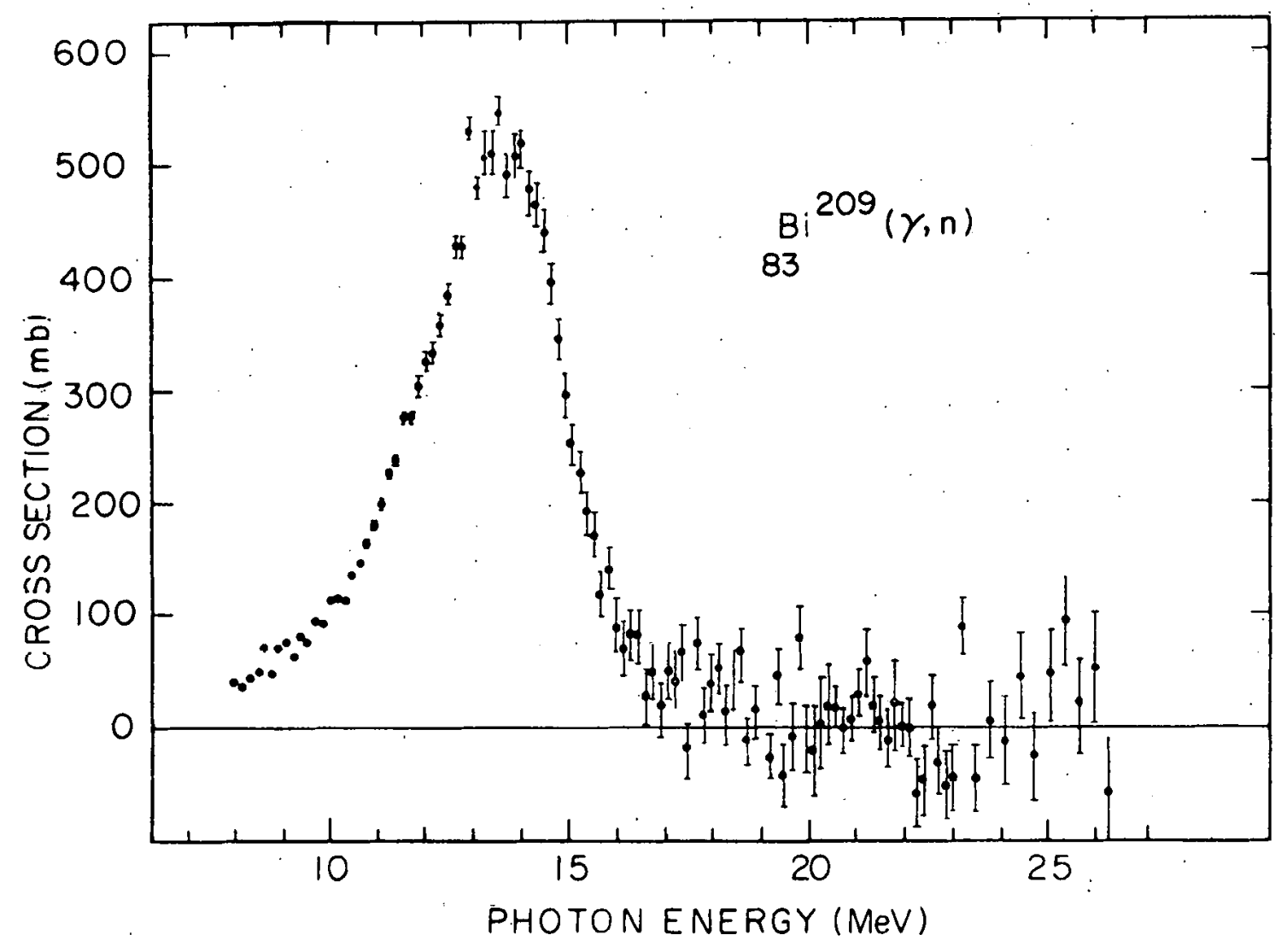


$61 a$

THIS PAGE

WAS INTENTIONALLY

LEFT BLANK 
Figure 19. The "uncorrected" $(\gamma, 2 n)$ cross section curve. 


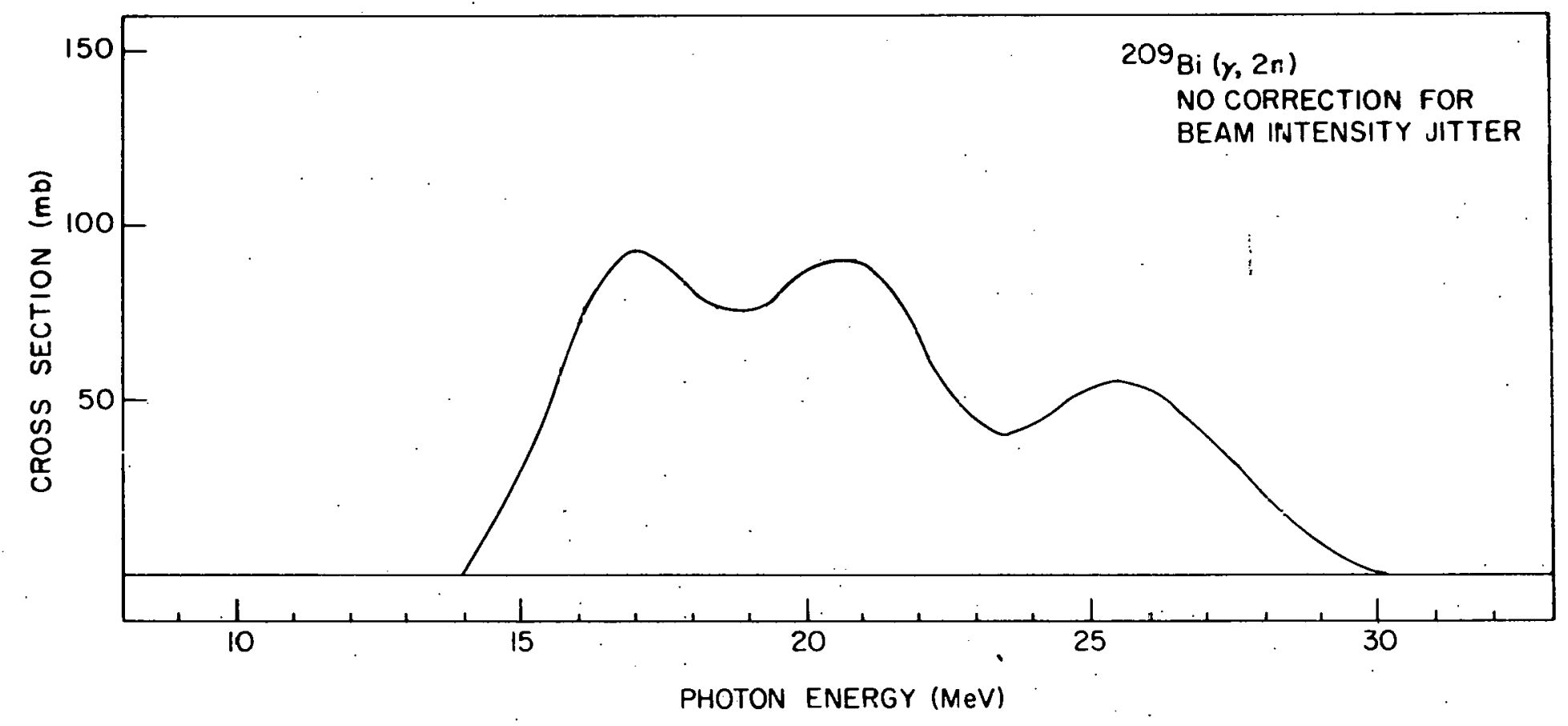




\section{THIS PAGE \\ WAS INTENTIONALLY \\ LEFT BLANK}


Figure 20. The "corrected" $(\gamma, 2 n)$ cross section curve. 


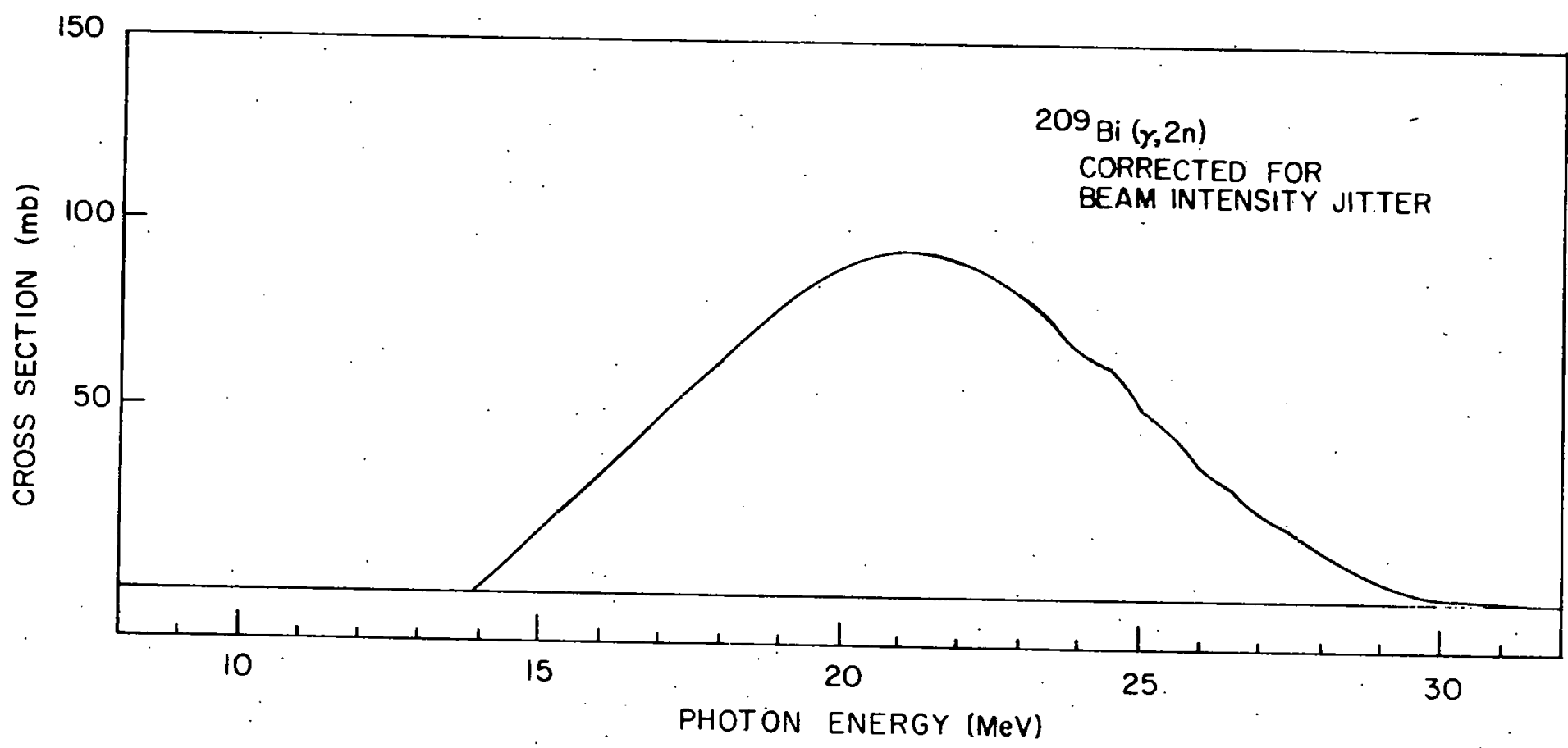

ڤิ 
$63 a$

THIS PAGE

WAS INTENTIONALLY

LEFT BLANK 
Fiqure 21. The $(Y, 2 n)$ cross section curve as obtained by Harvey et al. (17): 


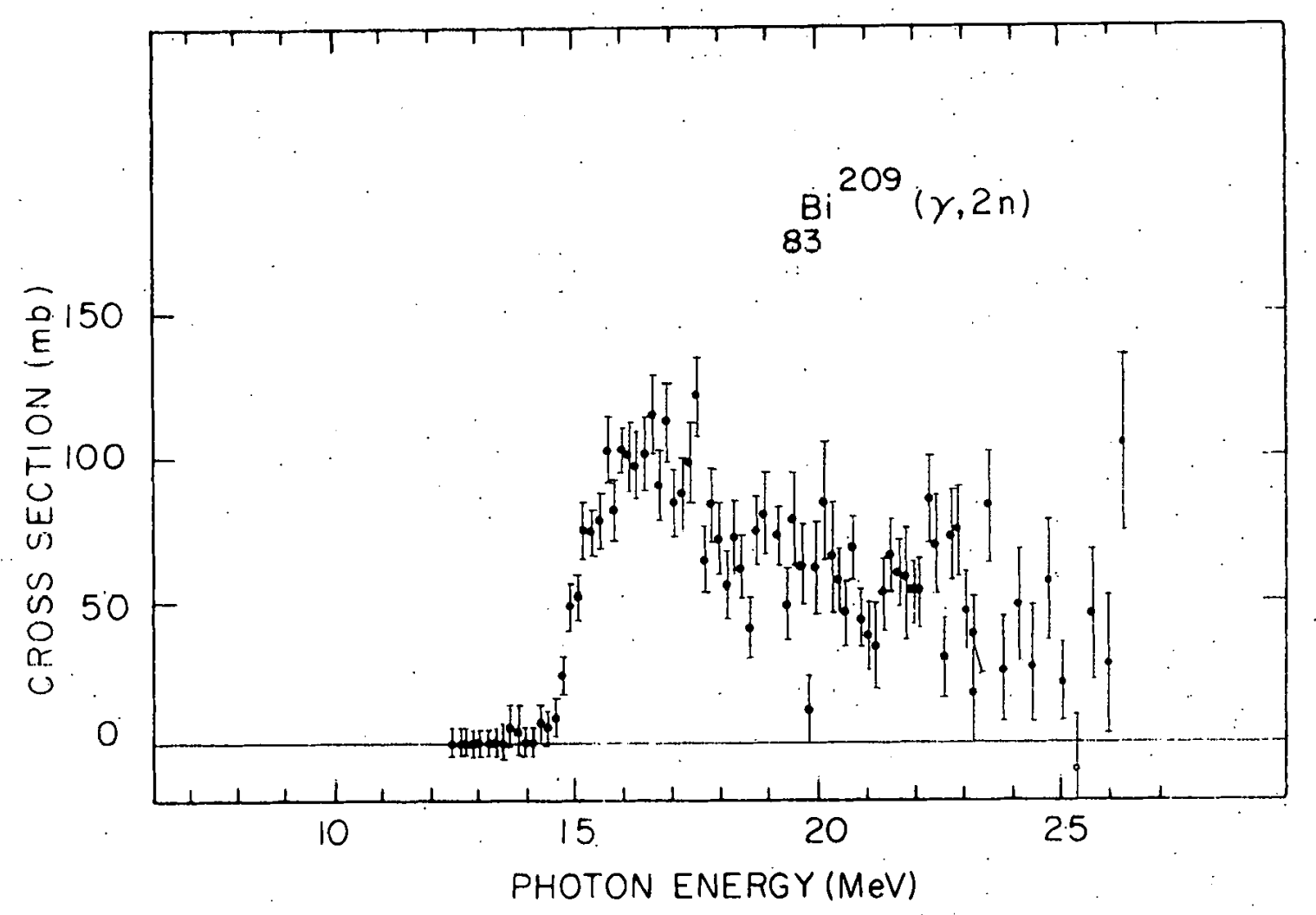


$64 a$

\section{THIS PAGE}

WAS INTENTIONALLY

LEFT BLANK 
Figure 22. The total absorption cross section curve as obtained in this experinent. The broken line is the Lorentz line with the parameters given in the text. 


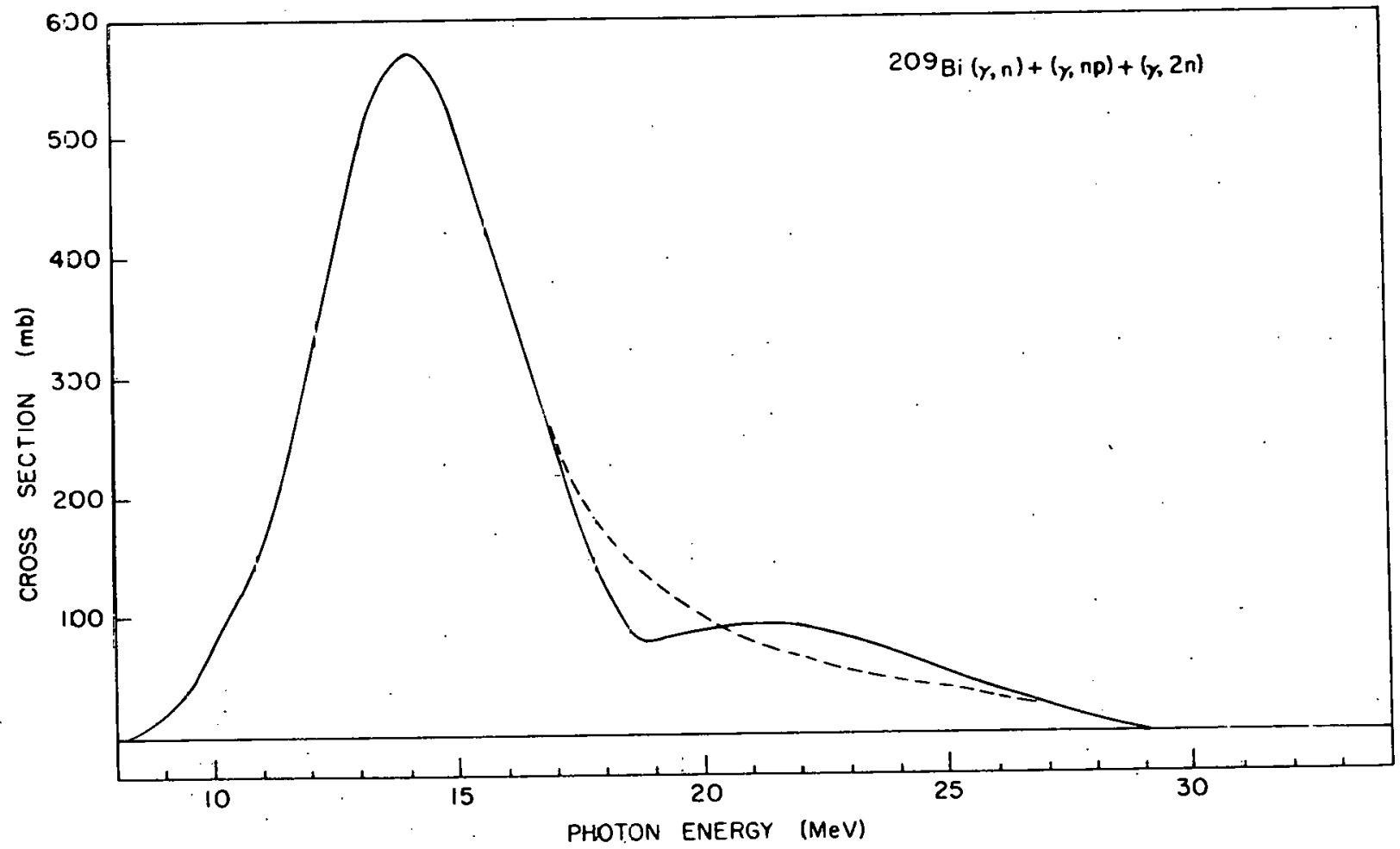

जิ 
age value reaained quite constant (less than $20 x$ uncertainty in the integrated cross section for $(y, 3 n)$ ). The wethod is explained in more detail in appendix I. The error assignment was estimated from the scatter of the points about the mean obtained at higher energies.

For the $(\gamma, 3 n)$ reaction, the value of the integrated cross section was $172 \pm 40 \mathrm{mb} \times$ Mer, and for $(y, 4 \mathrm{n})$ reaction $220 \pm 100 m b \times M e V$.

Wyckoff (36) measured the ratios of the yields for high nultiplicity photoneutron reactions using the bremsstrahlung with the end point energy of 137 HeV. From his data he estimates relative integrated cross sections. Using the results of Harvey et al. (17) for normalization, he gave the values for the integrated cross section for $(\gamma, 3 n)$ reaction as $168 \pm 25 \mathrm{mb} \times \mathrm{ber}$, and for $(\gamma, 4 \mathrm{n})$ reaction $88 \pm 22 \mathrm{mb} \times \mathrm{HeV}$.

Fiqure 22 shows the total photoneutron cross section as obtained in this experiment. The broken line shous the best fit to the Lorentz line vith the paraneters given above.

\section{B. Discussion}

The total integrated cross section (so sum rule) obtained in this experiment vas $3954 \pm 150$ ab xhev. This result is significantly higher than the classical sum rule yhich qives $s_{0}=3000 \mathrm{mb}$ weV. In fact, the $(\gamma, n)$ integrated cross section alone, alnost exhaust this sua rule. This suggests that there is sigaificant contribution of Majorana exchange 
force in the nuclear potential. The value obtained for parameter $\beta$ was $\beta=1.3$. Since this experiment extends only to $58 \mathrm{MeV}$. B must be considered a lower limit to its true value. No evidence was obtained for a reduction in total cross section at higher energies. If the cross section remains constant above $30 \mathrm{HeV}$, then another 1000 abxliev woula be obtained for $s_{n}$ between 60 and 140 Mev, corresponding to a $B$ of 1.65. Thus measurements to higher energies are clearly needed. Another important fact obtained from this experiment was that $(\gamma, 3 n)$ and $(\gamma, 4 n)$ reactions contribute quite significantly in the total cross section. Their total contribution up to 58 Mev was about $400 \mathrm{mbxheV}$ wich was slightly above $10 x$ of the total.

The measurement of the $s_{-1}$ sam rule gave the result $s_{-1}=263.6 \mathrm{ab}$, which gave the value of parameter $c$ in Levinger's formula $c=0.21 \pm 0.02 \mathrm{mb}$. This value agrees very well with the experimental results for $200 \mathrm{~Pb}$ and $197 \mathrm{Au}$ of veyssiere et al. (17) who obtained $c=0.20 \pm 0.02$ mb, and Lepretre et al. (37), for Rb, Sr, $99,90 \mathrm{Zr}$ and $93 \mathrm{Nb}$ whose value was $c=0.18 \pm 0.02 \mathrm{mb}$. These results, howerer, were about $30 x$ lower 3 han the theoretical predictions by Levinger who gave the values of $c=0.35 \mathrm{mb}$ for an isotropic harmonic potential, and $c=0.30 \mathrm{mb}$ for finite square well. An estimate was made of the contribution to the $s_{-1}$ sum rule assuming a constant cross section between 60 and $140 \mathrm{MeV}$. This estiuate 
gave $10 \mathrm{mb}$ which was less than $5 \%$ of the total. The $s_{-1}$ sum rule is proportional to the mean square of electric dipole operator. Mithout correlations in the nuclear wave function the dipole operator is proportional to the nuclear radius. An $s-1$ of $263.6 \mathrm{mb}$ corresponds to an estimate of $\left\langle r^{2}\right\rangle_{00} 30 \%$ smaller than the values deduced from high energy electron scattering. This fact suggests that the motion of nucleons is strongly anticorcelated inside the nucleus. (This anticorrelation may not come from the pauli principle, howerer. since the single slater determinants were used in the determination of the original value of mean square radius).

The measurement of the $s_{-2}$ sum rule gave the value $s_{-2}=16.6 \pm 1 \mathrm{mb} / \mathrm{MeV}$. The agreement with the result of Harvey et al. (16) who gave $s_{-2}=16.6 \pm 1.6 \mathrm{mb} / \mathrm{Hev}$, and theoretical. prediction of Migdal is excellent.

The results discussed above, are sumarized in Table 1.

\section{Summary}

The photoneutron cross sections for $209 \mathrm{Bi}$ had been measured using the direct neutron detection technique. By increasing the neutron, counting efficiency of the detector up to $76 \%$. it was possible to measure for the first time the absolute cross sections of high neutron wultiplicity reactions (up to $(Y, 4 n)$, for Bismuth. By employing on-line computer for the data collection and control of the experiment it vas possible to collect very large anount of data practically 
without human error. The technique for correcting the results for for the beam intensity instability has been developed. The experimental part of this method needs some improvement, hovever, especially in measuring the bean burst intensity.

The most serious limitation of the systen was the low repetition rate of the synchrotron. With the rate of 60 pulses/sec it took on average 2.5 hours to obtain one point on the yield curve.

- clear evidence for including exchange forces is cited as vell as evidence for nuclear correlation in the nucleus. 
Table 1. Integrated cross sections and sum rules for $209 \mathrm{Bi}$.

\begin{tabular}{|c|c|c|c|}
\hline References & 1 & 1 & I present work \\
\hline$E_{M}($ MeV $)$ & $\begin{array}{l}1 \\
1 \\
1\end{array}$ & $137^{*}$ & 1 \\
\hline$s(Y, n) \quad(m b \times M e V)$ & 2170 & 1 & $2830 \pm 100$ \\
\hline$s(\gamma, 2 n)$ (mb.xHeV) & 760 & $i$ & $732 \pm 30$ \\
\hline$s(\gamma, 3 n) \quad(m b \times H e V)$ & $i$ & $168 \pm 25$ & $172 \pm 40$ \\
\hline$s(r, 4 n) \quad(n b \times H e V)$ & 1 & $88 \pm 22$ & $220 \pm 100$ \\
\hline$s(\gamma, t o t)(a b \times M \in V)$ & $2930 \pm 290$ & $i$ & $3954 \pm 150^{* *}$ \\
\hline $60 \times N Z / A \quad(m b \times M e V)$. & 3000 & 3000 & 3000 \\
\hline$s-1 \quad(m b)$ & 1 & i & $263.6 \pm 25$ \\
\hline $\mathrm{s}-2 \quad(\mathrm{mb} / \mathrm{HeV})$ & $16.6 \pm 1.7$ & $i$ & $16.6 \pm 1.0$ \\
\hline
\end{tabular}

*) In this case the measurement was performed for one energy only. The cross sections were deduced from the ratio of the yields assuming the absorption. **) Error obtained from quadrature. 
VII. LITERATURE CITED

1. C.C. Jones, Thesis, Iowa State Oniversity of Science and Technology. Ames, 1967 (unpublished).

2. J.C. Levinger, Ann. Bev. Nucl. Sci. 4. 13 (1954).

3. D.H. Milkinson, Ann. Rev. Nucl. Sci. 9.1 (1959).

4. M. Danos and E.G. Fuller, Ann. Nucl. Sci. 15. 29 (1965)

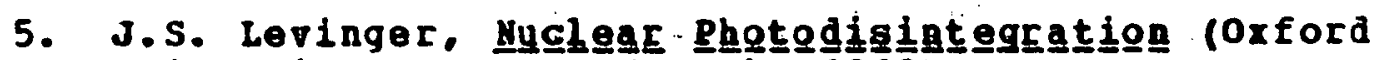
University Press, Nevi York, 1960 ).

6. B. Hayuard, Rev. Mod. Phys. 35. 324 (1963) .

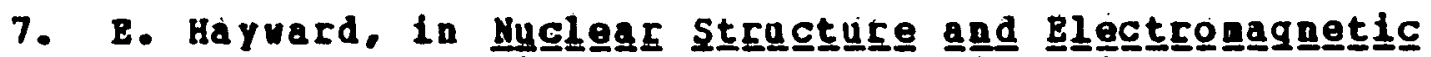
Interactions, edited by $\mathrm{N}$. MacDonald (Plenun press. Nev York. 1965), PP. 141-209.

8. B. S. Spicer, Nuovo Cimento Suppl. 2. 243 (1964).

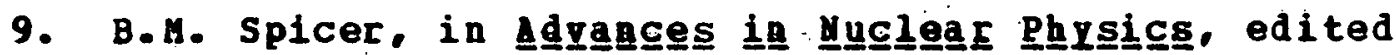
by. . Baranger and $B$. Vogt (PIenum Press. New York. 1969). Vol 2.

10. 0.V. Bogdankevich and P.A. Nikolaev. Methogods in Breges Etrahligung Regeacrch (Academic Press, New York, 1966).

11. B.I. Gorgachev, Atonic Energy Rev. 2. No. 3, 71 (1964).

12. B.C. Cook, R.C. Morrison and F.H. Schamber, Phys. Lett. 25. 685 (1970).

13. V. Gillet, A.M. Green and. B.A. Sanderson, Nucl. Phys. 88. $321(1966)$.

14. V.V. Balashov and N.4. Kabachnik, Phys. Lett. 6, 316 (1967) .

15. R.F. Barrett and P.P. Delsanto, Hucl. Phys. A173. 641 . (1971).

16. A. Veyssiere, H. Beil, R. Bergere, R. Carlos and

A. Lepretre, Nucl. Phys. A159; 561 (1970). 
17. R.B. Harvey: J.T. Caldvell, B.I. Bramblett and S.C. Pultz Phys. Rev. 136, B 126 (1964).

18. A, Higdal, J.* Phys., OSSR B., 331 (1944).

19. M. Goldhaber and E, Teller, Phys. Rev. 74, 1046 (1948).

20. J.H. Blatt and V.F. Meisskopf. Thegretigal Leclear Physilcㅗㅇ, J. Hiley, Mex York (1952).

21. B.G. Johnson. Thesis, Iowa state University of Science and Technology, Anes 1970, (unpublished).

22. S. Costa, Nucl. Instr., Methods 2.1. 129 (1963).

23. B.C. Cook and C. Jones, Nucl. Instr. Hethods 59 . $229(1968)$.

24. B.I. Goryachev, Atomaya Energiya 12, 246 (1962).

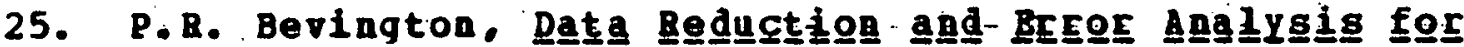

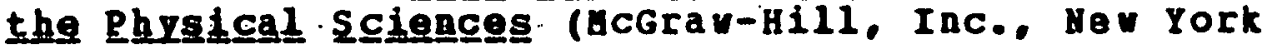
1969). Chapter II.

26. D. H. Marquardt, J. Soc. Ind. Appl. Math. 11, 431 (1963).

27. J.E. Griffin. Thesis, Iova State Onirersity of Science and Technology. Ames, 1963 (unpublished).

29. J.S. Pruitt and S.R., Donen, WBS Report 6218, (1958).

30. G. E. Clark, Private comanication

31. A. De Volpl and K.G. Porges, Phys. Ber., C1. 683 (1970).

32. L.I. Schiff, Phys. Rev, 8 3 , 252 (1951).

33. A.S. Penfold and J.B. Leiss, Phys. Bev..114, 1332 (1959).

34. B.C. Cook, Nucl. Instr. Hethods 24, 256 (1963).

35. E. Bramanis, T.K. Deague, R.S. Hicks, R.J. Rughes, E.G. Buirhead, R.H. Sambell and R.J.J., Steward, Nucl. Instr. Hethods 109. 59 (1972).

36. J.H. Nyckoff, Phys. Rev. 159, 963 (1967).

37. A. Lepretre, H. Bell, R. Bergere, P. Carlos and A. Veyssiere, Nucl. Phys. $4175 ; 609$ (1971). 
VIII. ACKNORLEDGHENTS

I wish to express ay gratitude to any people whose interest, encouragement, sugqestions and participation ade this work possible. In particular the contribution of the following people are greatly appreciated:

My mator professor, Dr. B.C. Cook for his interest, encouragement and support throughout my graduate career. Dr. J.E.E. Baglin whose any suggestions and discussions have contributed greatly to the success of this vork. Special thanks go to Dr. F.H. Schamber whose cooperation and contribution in writing most of the programs for the $S_{D S}$ conputer made this project possible. Mr. George Holland uhose help with the experinental details and wo along with Mr. Bill Glass and Dr*A*J・-Bureau kept synchrotron and experimental equipment operational. Mr. Bob pritchard, Mr. Jon Mccarthy. Dr. Gary Clark, Dr.Harold vandermolen, Mr. Dave Promme, Mr. Gerry Keith and Mr. Charles Gale for their help in operating the synchrotron and collecting the data. 


\section{APPENDIX I}

Let us rewrite Equation 34 in a vector form:

$$
\overrightarrow{\mathbf{Y}}=\mathrm{N} \cdot \overrightarrow{\mathbf{S}}=\mathrm{N} \cdot \mathrm{D} \cdot \overrightarrow{\mathbf{S}}
$$

where $\vec{S}$ is a vector of the integrated cross sections, that is $\vec{S}_{j}$ is integrated cross section ap to energy $E_{j}$, and matrix $D$ is defined as:

$$
D=\left(\begin{array}{rrrr}
1 & & & \\
-1 & 1 & & \\
0 & -1 & 1 & \\
0 & 0 & -1 & 1 \\
\cdots & \ldots & \ldots & \ldots
\end{array}\right)
$$

Therefore

$$
\begin{array}{ll}
\vec{S} & =(N \cdot D)^{-1} \cdot \vec{Y}=D^{-1} \cdot N^{-1} \cdot \vec{Y} \\
\text { or } \quad \vec{S} & =D^{-1} \cdot \vec{\sigma}
\end{array}
$$

but

$$
D^{-1}=\left(\begin{array}{cccc}
1 & & & \\
1 & 1 & & \\
1 & 1 & 1 & \\
1 & 1 & 1 & 1 \\
\cdots & \ldots & \ldots & \ldots
\end{array}\right)
$$

So in order to obtain the integrated cross section, all ve have to do is to sum all the values of the differential cross section. 
In practice, hovever, the measured yields have an error $\delta \vec{Y}$. therefore

$$
\vec{S}=D^{-1} \cdot N^{-1} \cdot(\vec{Y}+\delta \vec{Y})
$$

If we measure the average over large number of measurements:

$$
\langle\vec{S}\rangle=\left\langle D^{-i} \cdot \vec{\sigma}\right\rangle+D^{-1} \cdot N^{-1} \cdot\langle\delta \vec{Y}\rangle \rightarrow\left\langle D^{-1} \cdot \vec{\sigma}\right\rangle
$$

we indeed will approach the true value of the integrated cross section with no bias. Let us now find the error matrix of the integrated cross section:

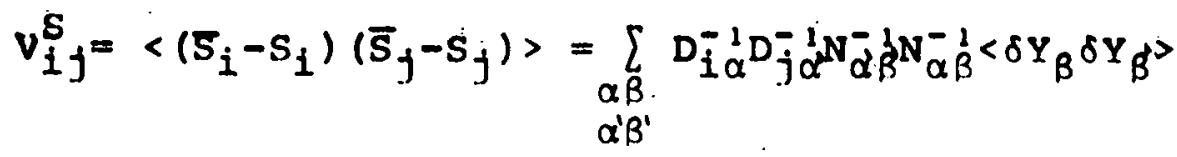

$$
\begin{aligned}
& =\sum_{\alpha \beta} D_{i \alpha^{\prime}}^{-1} D_{j}^{-1} \alpha^{-1} N_{\alpha \beta}^{-1} N_{\alpha^{\prime} \beta}^{-1} E_{\beta}^{2}
\end{aligned}
$$

since

$$
\left\langle\delta Y_{R} \delta Y_{B^{\prime}}\right\rangle=\varepsilon_{B}^{2} \delta B^{\prime}
$$

now the matrix

$$
v_{\alpha \alpha 1}^{s}=\sum_{\beta} N_{\alpha \beta}^{-1} N_{\alpha+\beta}^{-} \varepsilon_{\beta}^{2}
$$

is an error watrix of a differential cross section $\vec{s}$. For the bremsstrahlung experiment this matrix is strongly anticorrelated, that is, the elements are oscillating very strongly between positive and negative values of about same magnitude. The error matrix for the integrated cross section 
is given by:

$$
v_{i j}^{S}=\sum_{\alpha \alpha} D_{i}^{-1} D_{j}^{-1} v_{\alpha \alpha}^{\sigma}
$$

since the nultiplication by a matrix $D$ is effectively the addition of elenents of matrix $v^{s}$, the elements of matrix $v^{s}$ wil be very small compared to the elements of $v^{3}$. That means, that despite having large errors in estimating the values of a differential cross section, the error of the integrated cross section obtained by adding the values of differential cross section. vill be very small. That also means that if we calculate the average values of the integrated cross section over several energy intervals, the resultant values vill differ very little.

As an ilustration let us assume that the bremstrahlung has a constant value up to the end point energy. The $N$ matrix can be written as:

$$
\begin{aligned}
& N_{i j}=\xi \text { if } j \leq i \\
& N_{i j}=0 \text { if } j>i
\end{aligned}
$$

Notice that $N=D^{-1} \cdot \xi$

The $v^{s}$ matrix will be then: $(\xi=1)$

$$
\mathbf{v}^{\mathbf{s}}=\left(\begin{array}{cccc}
1 & 0 & 0 & 0 \\
-1 & 1 & 0 & 0 \\
0 & -1 & 1 & 0 \\
0 & 0 & -1 & 1 \\
\ldots & \ldots & \ldots & 0
\end{array}\right)\left(\begin{array}{lllll}
\varepsilon_{1}^{2} & & & \\
& \varepsilon_{3}^{2} & & \\
& & \varepsilon_{3}^{2} & \\
& & & \varepsilon_{4}^{2}
\end{array}\right)\left(\begin{array}{cccc}
1 & -1 & 0 & 0 \\
0 & 1 & -1 & 0 \\
0 & 0 & 1 & -1 \\
0 & 0 & 0 & -1 \\
\ldots & \ldots & \ldots & \ldots
\end{array}\right)
$$




$$
=\left(\begin{array}{cccc}
\varepsilon_{1}^{2} & \varepsilon_{1}^{2} & 0 & 0 \\
-\varepsilon_{1}^{2} & \varepsilon_{1}^{2}+\varepsilon_{2}^{2} & -\varepsilon_{2}^{2} & 0 \\
0 & -\varepsilon_{2}^{2} & \varepsilon_{2}^{2}+\varepsilon_{3}^{2} & -\varepsilon_{3}^{2} \\
0 & 0 & \div \varepsilon_{3}^{2} & \varepsilon_{3}^{2}+\varepsilon_{4}^{2}
\end{array}\right)
$$

The elements on the wain diagonal are the errors in differential cross section. They are bigger then the errors in the vield. If nov we obtain the $v^{s}$ matrix:

$$
\begin{aligned}
& V^{S}=\left(\begin{array}{llll}
1 & 0 & 0 & 0 \\
1 & 1 & 0 & 0 \\
1 & 1 & 1 & 0 \\
1 & 1 & 1 & 1
\end{array}\right)\left(\begin{array}{cccc}
-\varepsilon_{1}^{2} & \varepsilon_{1}^{2} & 0 & 0 \\
-\varepsilon_{1}^{2} & \varepsilon_{1}^{2}+\varepsilon_{2}^{2} & -\varepsilon_{2}^{2} & 0 \\
0 & -\varepsilon_{2}^{2} & \varepsilon_{2}^{2}+\varepsilon_{3}^{2} & -\varepsilon_{3}^{2} \\
0 & 0 & -\varepsilon_{3}^{2} & \varepsilon_{3}^{2}+\varepsilon_{4}^{2}
\end{array}\right)\left(\begin{array}{llll}
1 & 1 & 1 & 1 \\
0 & 1 & 1 & 1 \\
0 & 0 & 1 & 1 \\
0 & 0 & 0 & 1
\end{array}\right) \\
& =\left(\begin{array}{llll}
\varepsilon_{1}^{2} & & & \\
& \varepsilon_{2}^{2} & & \\
& & \varepsilon_{3}^{2} & \\
& & & \varepsilon_{4}^{2}
\end{array}\right)
\end{aligned}
$$

The matrix is the same as for the yield. (It is not surprizing since for this kind of bremsstrahlung the yield is fust the integrated cross section). The errors are reduced and same as in the yield. For the real bremsstrahlung ve can therefore expect that the errors will be of the order of the errors in the pield. 